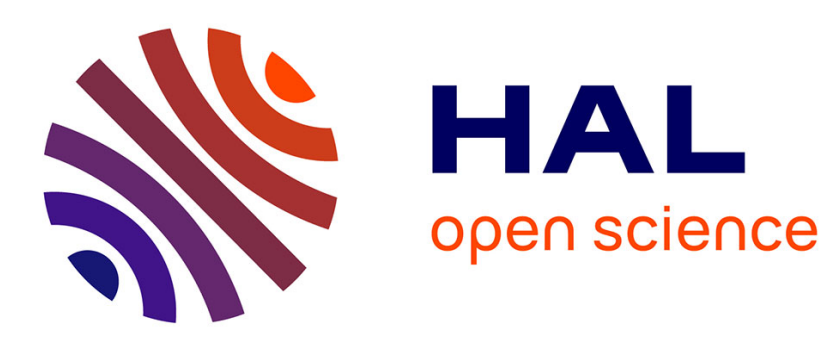

\title{
Isolated cortical computations during delta waves support memory consolidation
}

\author{
Ralitsa Todorova, Michaël Zugaro
}

\section{To cite this version:}

Ralitsa Todorova, Michaël Zugaro. Isolated cortical computations during delta waves support memory consolidation. Science, 2019, 366 (6463), pp.377-381. 10.1126/science.aay0616 . hal-02331039

\section{HAL Id: hal-02331039 \\ https://hal.science/hal-02331039}

Submitted on 31 Oct 2019

HAL is a multi-disciplinary open access archive for the deposit and dissemination of scientific research documents, whether they are published or not. The documents may come from teaching and research institutions in France or abroad, or from public or private research centers.
L'archive ouverte pluridisciplinaire HAL, est destinée au dépôt et à la diffusion de documents scientifiques de niveau recherche, publiés ou non, émanant des établissements d'enseignement et de recherche français ou étrangers, des laboratoires publics ou privés. 


\title{
Isolated cortical computations during delta waves
}

\section{support memory consolidation}

\author{
Ralitsa Todorova, ${ }^{1}$ Michaël Zugaro, ${ }^{1 *}$ \\ ${ }^{1}$ Center for Interdisciplinary Research in Biology (CIRB), \\ Collège de France, CNRS, INSERM, Université PSL, Paris, France \\ ${ }^{*}$ Corresponding author. E-mail: michael.zugaro@college-de-france.fr
}

\begin{abstract}
Delta waves have been described as periods of generalized silence across the cortex, and their alternation with periods of endogenous activity results in the slow oscillation of slow wave sleep. Despite evidence that delta waves are instrumental for memory consolidation, their specific role in reshaping cortical functional circuits remains puzzling. We found that delta waves are not periods of complete silence, and the residual activity is not mere neuronal noise. Instead, cortical cells involved in learning a spatial memory task subsequently formed cell assemblies during delta waves in response to transient reactivation of hippocampal ensembles during ripples. This occurred selectively during endogenous or induced memory consolidation. Thus, delta waves represent isolated cortical computations tightly related to ongoing information processing underlying memory consolidation.
\end{abstract}

Most of our time spent asleep is dominated by slow oscillations $(0.1-1 \mathrm{~Hz})$, when cortical neurons synchronously alternate between a depolarized (up) state associated with high levels of endogenous activity, and a hyperpolarized (down) state when neurons remain silent (1). Delta waves are large deflections of the local field potential (LFP) which correspond to the down states of the slow oscillation, and are thus considered periods of generalized cortical silence. The slow oscillation plays a causal role in memory consolidation (2-5), in particular by orchestrating an information flow between the hippocampus and the neocortex (6). Indeed, delta waves tend to occur closely before or after hippocampal ripples (7), which are instrumental for memory consolidation $(8,9)$. Hippocampal replay of awake activity (10), biased by inputs from sensory cortices $(11,12)$, initiates reactivation of prefrontal cortical cell assemblies $(13,14)$ just before the occurrence of a delta wave (7). Cortical synaptic plasticity subsequently takes place during network reorganization early in the following up state $(15,16)$ and during massive calcium entry accompanying the ensuing sleep spindle (17-19). This hippocampocortical dialogue (20-22) is instrumental for memory consolidation (5). However, the incursion of generalized silence (delta wave) precisely between periods of information exchange and periods of network plasticity remains puzzling.
We recorded prefrontal cortical activity in 9 rats during slow wave sleep (5). Consistent with previous reports, most delta waves were accompanied by neuronal silence. Yet, occasionally spikes did occur during delta waves (Fig. 1A), and when considering cumulative spiking activity over all recorded delta waves, unexpected residual activity appeared at the very peak of the waves (Fig. 1B-C; spike waveforms recorded during delta waves were not distinguishable from spike waveforms recorded outside delta waves, Fig. S1). Upon closer examination, neuronal activity occurred consistently in a substantial fraction of delta waves $(12 \%)$, where one or a few neurons remained active while the rest of the population became silent (Fig. 1D). We call this unexpected persisting activity 'delta spikes'. To investigate if delta spikes were restricted to a particular subset of neurons, we counted the number of delta waves in which each unit emitted one or more spikes. As it happened, every single recorded unit fired during delta waves, suggesting instead that persisting firing may actually constitute a widespread phenomenon (Fig. 1D, Fig. S2).

We then wondered if delta spikes tended to occur in specific delta waves with distinct characteristics. We thus compared delta waves in which we did or did not detect cortical spikes, and found no significant difference between the two groups in terms of waveform (Fig. 1E), duration, timing (Fig. S3), 


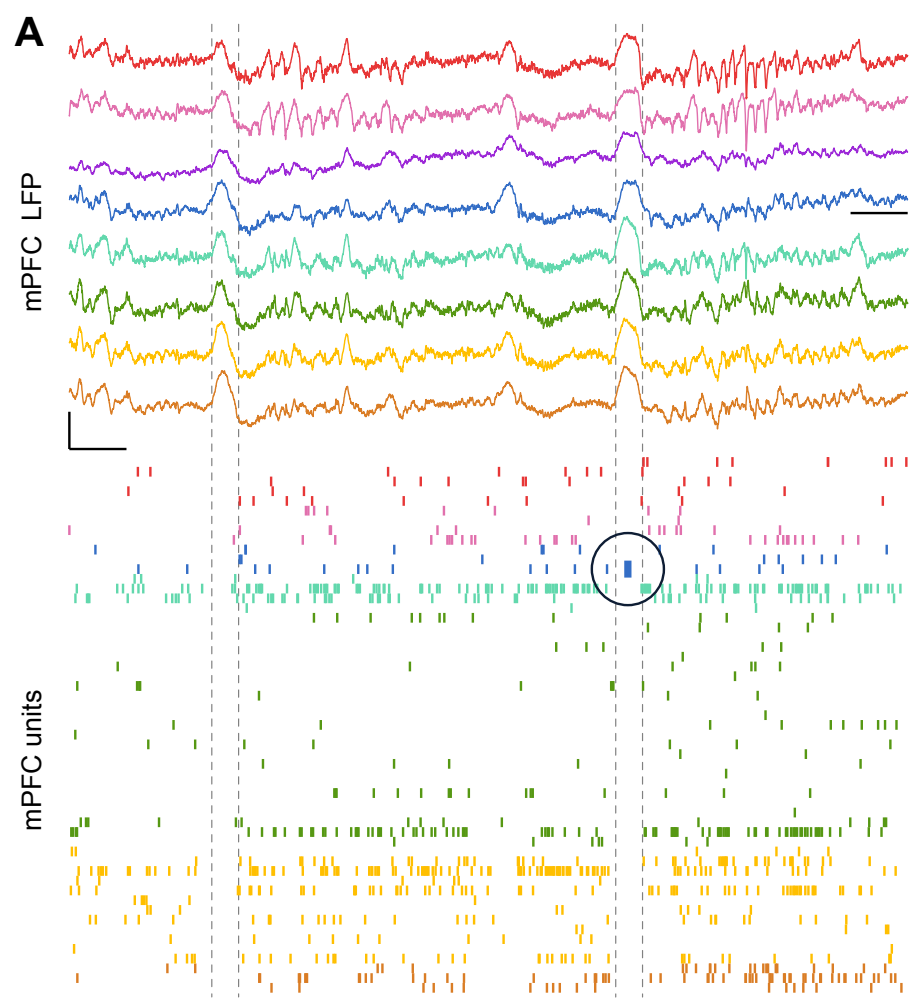

B

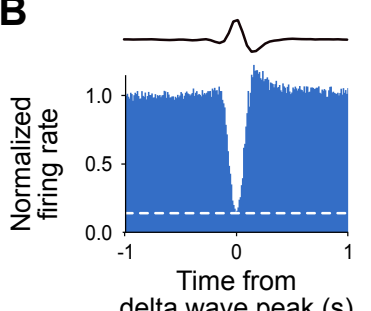

D
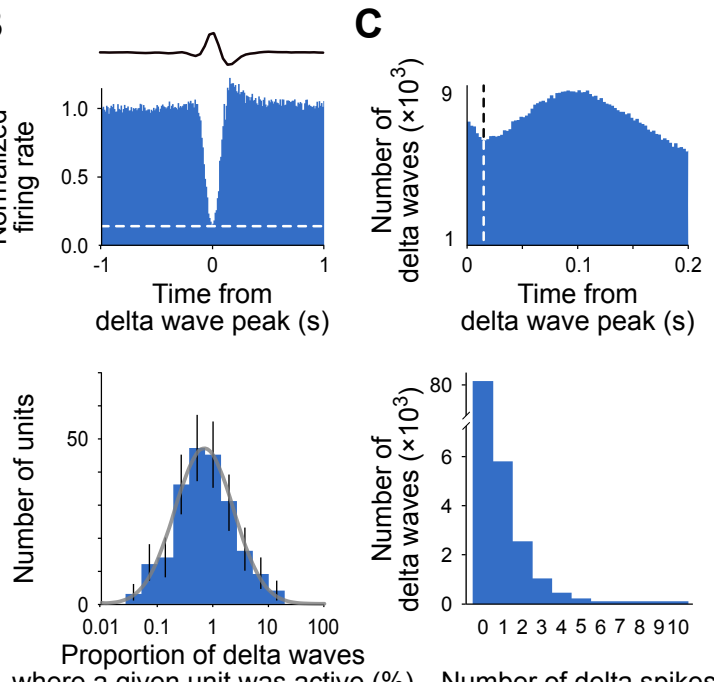

delta wave peak (s)

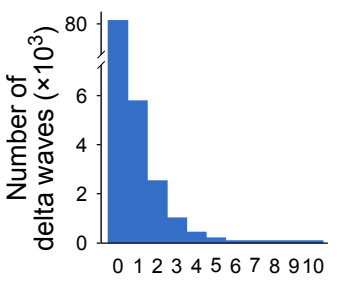

where a given unit was active (\%) Number of delta spikes

E

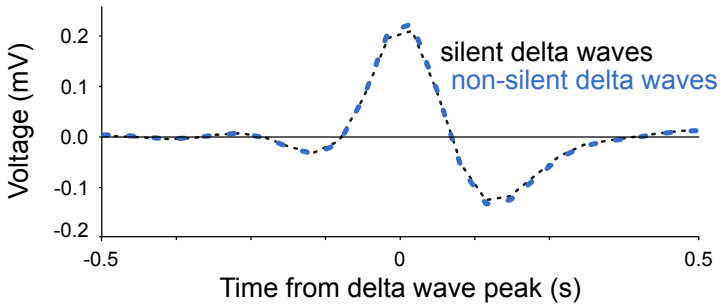

Fig. 1. Delta spikes. (A) Example of a non-silent delta wave. Colored curves: local field potentials recorded from the medial prefrontal cortex (color: recording channel). Colored vertical ticks: spikes emitted by simultaneously recorded prefrontal units (color: channel from which the unit was recorded). Dashed lines indicate the beginning and end of delta waves. Note the delta spike (black circle) during the second delta wave, when the rest of the network remains silent. Black calibration bars: $0.5 \mathrm{~s} ; 1 \mathrm{mV}$. (B) Mean peri-event time histogram (PETH) of the normalized firing rate of prefrontal units centered on delta waves (top curve: mean field event). Note residual activity (dashed white line) during delta waves. (C) Time distribution of the spikes emitted by each prefrontal neuron closest to each delta wave. The large peak at $\sim 100$ ms corresponds to activity in the up state. The smaller peak consists of spikes occurring during delta waves. Dashed line: $15 \mathrm{~ms}$ upper threshold used to define delta spikes in subsequent analyses (all results were confirmed using $\pm 30 \mathrm{~ms}$ time windows). (D) Left: number of units that discharged in a given proportion of delta waves (gray curve: log-normal fit with the same mean and variance as the data; error bars: 95\% confidence intervals from bootstrapped data). No unit fired in $0 \%$ of the delta waves. Right: number of delta waves containing a given number of delta spikes. (E) No difference in average waveforms between silent (black, $n=101,161$ ) and non-silent (blue, $n=12,205$ ) delta waves (Monte-Carlo test, $P>0.05$ ).

depth (Fig. S4), decreased gamma power, or coupling with hippocampal ripples and thalamocortical spindles (Fig. S5). This suggests that spikes could take place during virtually all delta waves, but may remain undetected given the limited number of recorded neurons relative to the entire population (Fig. S6). We thus hypothesize that firing during delta waves might be an overlooked phenomenon manifested in possibly all delta waves.

These findings indicate that during any given delta wave, the cortical network becomes silent, except for a small but ever-changing minority of cells. The most parsimonious explanation would be that delta spikes constitute random activity reflecting imperfect coordination in the cortical alternation between up and down states. Yet, an alternative intriguing possibility is that this activity actually serves a well-defined computational function. A hallmark of cortical computation is the emergence of cell assemblies. We thus tested for the presence of recurring co-active cell ensembles, using two complementary approaches. As a first approach, we performed a standard independent component analysis (23), which identified multiple significant components active during delta waves (Fig. S7A,B). However, these components were likely to actually combine multiple smaller but overlapping cell ensembles, given the limited number of neurons 
active in any given delta wave. We thus performed a second analysis, to examine cooperative activity ('peer prediction', (24)) among delta spikes, an idiosyncratic property of cell assemblies. This showed that the delta spikes of one neuron could be predicted from the delta spikes of other neurons (Fig. S7C).
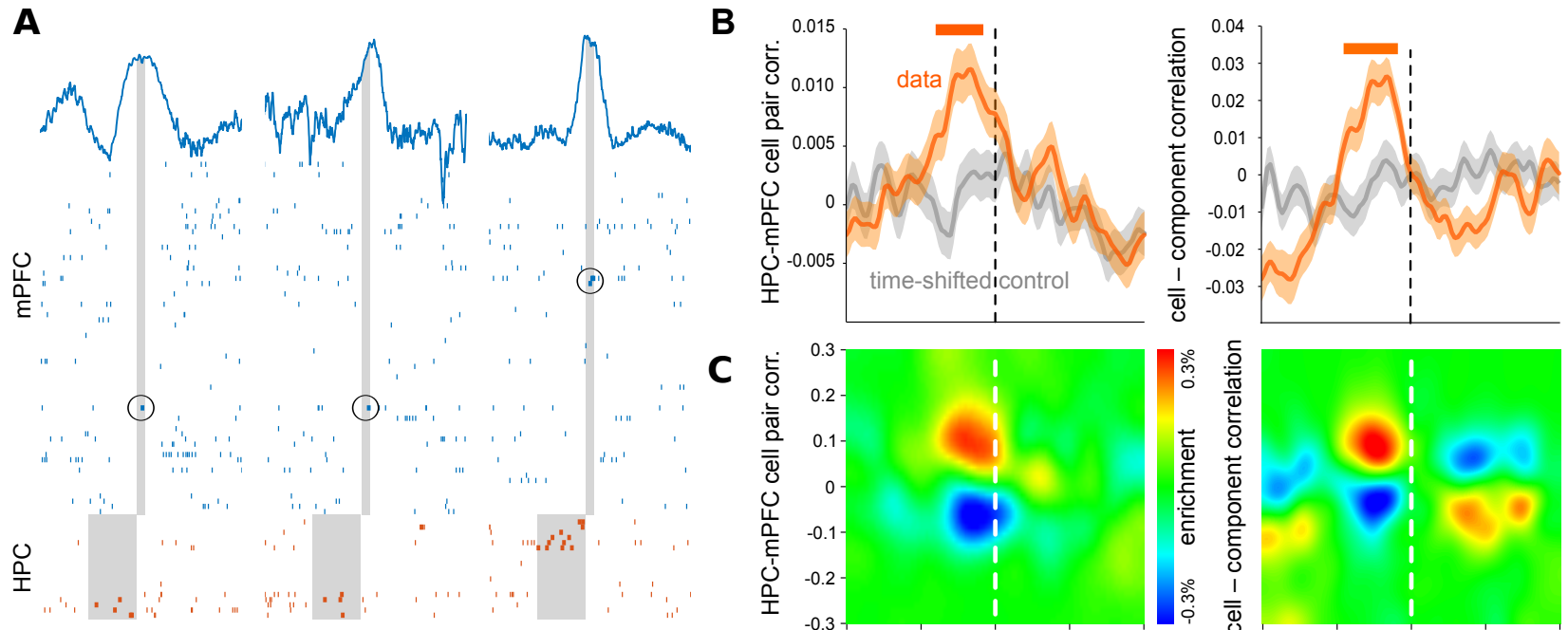

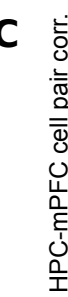
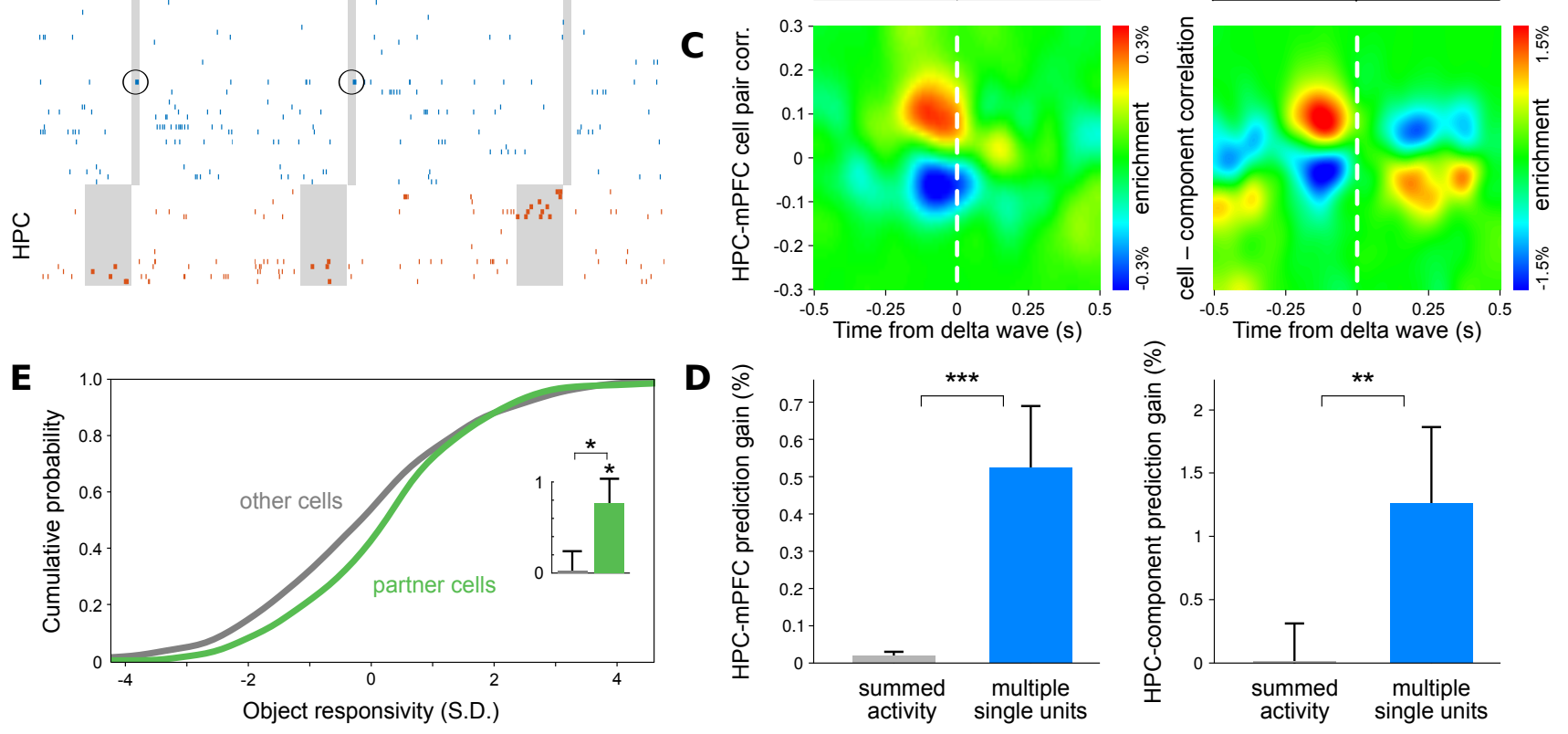

Fig. 2. Hippocampal ripple activity predicts delta spikes. (A) Delta spikes and preceding hippocampal activity. Blue traces: local field potentials from the medial prefrontal cortex. Colored ticks: simultaneously recorded prefrontal (blue) and hippocampal (red) spikes. Black circles indicate delta spikes emitted within $\pm 15 \mathrm{~ms}$ of delta wave peak (shaded area). In the first two delta waves, delta spikes were recorded from the same unit following similar hippocampal activity patterns. (B) Cross-correlations (curves and shaded areas, mean \pm s.e.m.; orange, data; grey, time-shifted control) between hippocampal ripple activity (sliding window) and delta spikes (fixed, $0 \mathrm{~s}$ ). Horizontal orange line: Monte-Carlo test, $P<0.05$. (C) Enrichment in positive correlations (comparative distribution between data and control in (B)) when hippocampal activity was correlated to subsequent prefrontal delta spikes. (D) Performance of a GLM trained to predict prefrontal activity during delta waves based on preceding hippocampal ripple activity (200 ms window). Delta spikes as well as delta components were significantly predicted by multiple single-unit hippocampal activity $(P=0.0403$ for delta spikes and $P=0.0052$ for delta components, Wilcoxon rank-sum test), but not by global hippocampal drive ignoring cell identity (summed activity, $P=0.2597$ for delta spikes and $P=0.3258$ for delta components, Wilcoxon rank-sum test). ${ }^{* *} P<0.01,{ }^{* * *} P<0.001$, Wilcoxon signed rank tests. (E). Object responsivity index for partner (green) vs other (grey) prefrontal units (curves: cumulative distributions; inset: mean \pm s.e.m.). Only partner prefrontal units showed positive object responsivity (partner units, $P=0.0162$; other units, $P=0.5967$, Wilcoxon signed rank test; partner vs other units, $P=0.0465$, Wilcoxon rank-sum test).

We then asked if delta spikes were involved in the hippocampo-cortical dialogue underlying memory consolidation. Because delta waves typically take place precisely between hippocampal replay and cortical reorganization for memory consolidation, this hypothesis would be expected to have two implications: 1) hippocampal activity during ripples should predict which neurons (or which assemblies) 
are active during the following delta wave, 2) this predictive bias should emerge following behavior, and predictable cortical cells should be involved in the reactivation of waking experience.
Rats were trained on a spatial memory task, and hippocampal and cortical activity was recorded both during behavior and subsequent memory consolidation during the first two hours of sleep (5).
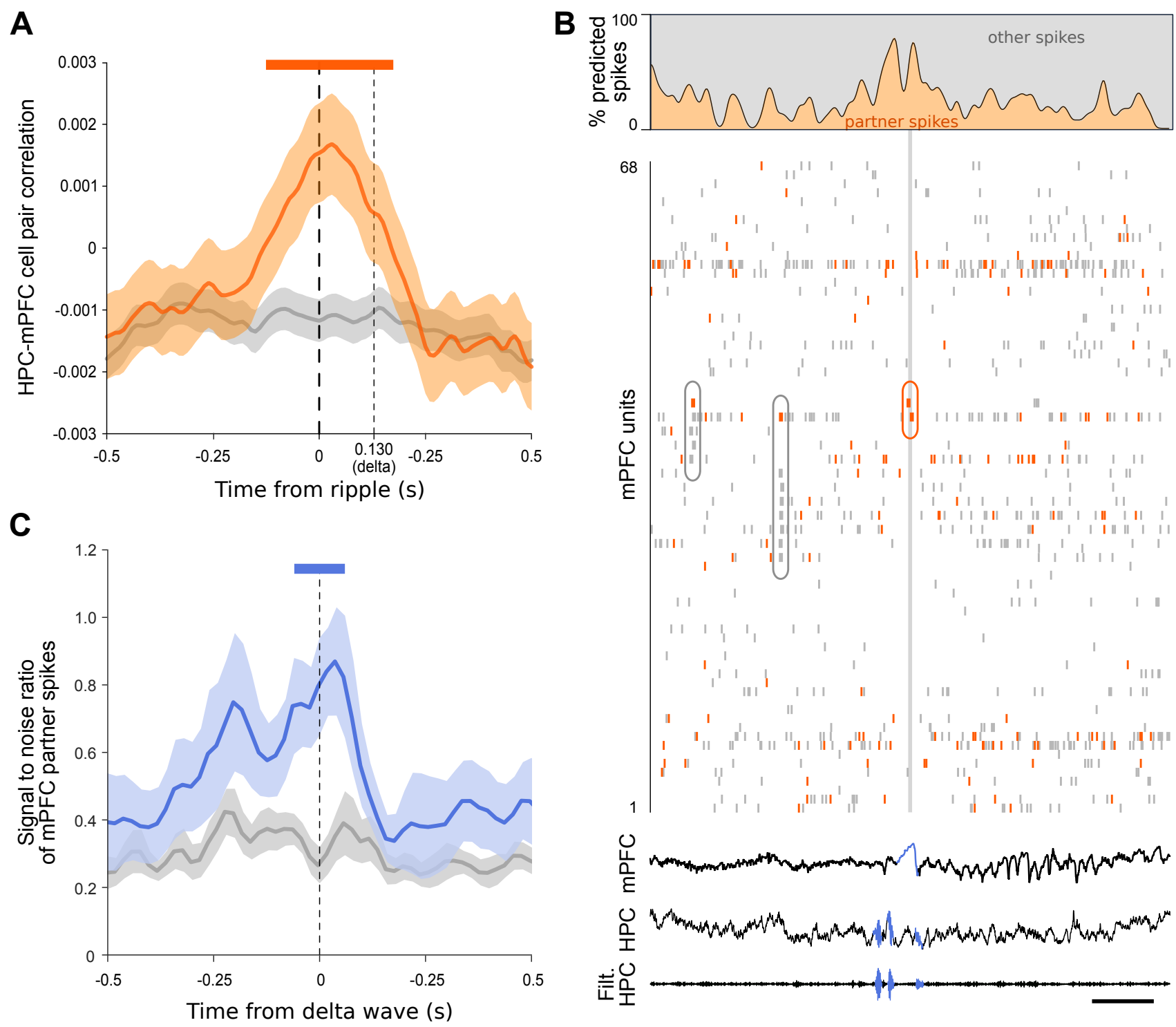

Fig. 3. Delta waves isolate cortical computations. (A) Cross-correlations (curves and shaded areas, mean \pm s.e.m.) between hippocampal ripple activity (fixed, $0 \mathrm{~s}$ ) and prefrontal activity (sliding window). Observed crosscorrelations (orange) were significantly different from a time-shifted control (grey) for cortical activity following ripples (horizontal orange line: Monte-Carlo test, $P<0.05$ ). Delta waves (dashed line, peak occurrence rate $130 \mathrm{~ms}$ after ripples) tend to occur within the critical window where prefrontal activity remains correlated with the preceding ripple activity. (B) Simultaneous recording of prefrontal and hippocampal activity around a delta wave (grey shaded rectangle). Top: proportion of prefrontal spikes predicted by the firing of hippocampal cells (partner spikes). Center: raster plot of spikes emitted by 68 simultaneously recorded prefontal units (red ticks: partner spikes, grey ticks: other spikes). Bottom: simultaneously recorded local field potentials in the mPFC (blue: delta wave) and hippocampus (broadband and ripple-band filtered signal; blue: ripples). During delta waves, partner spikes occurred in isolation (red ellipse). Partner spikes emitted by the same units outside delta waves (grey ellipses) formed a considerably smaller proportion of the ongoing cortical activity. Black calibration bar: 0.5 s. (C) Signal-to-noise ratio (curves and shaded areas, mean \pm s.e.m.) of partner spikes relative to other spikes around delta waves. Observed values (blue) were significantly different from a time-shifted control (grey) during delta waves (horizontal blue line: Monte-Carlo test, $P<0.05$ ). 
Hippocampal spiking activity during ripples was significantly correlated with immediately (50-200 ms) following cortical delta spikes (Fig. 2A-B; the effect was not due to data recorded from any single rat, Figs. S8). This increased correlation was due to a large proportion of positively correlated inter-regional pairs of neurons (Fig. 2C). Furthermore, ripple spikes were better correlated with delta spikes than with spikes occurring at similar delays during an up state (i.e. correlations were greater in the presence of a delta wave, Fig. S9). A generalized linear model (GLM) analysis showed that ensemble activity in the hippocampus could significantly predict which cortical cells would emit delta spikes (Fig. 2D). In contrast, delta spikes could not be predicted from the combined activity of all hippocampal units that ignored cell identity (multiunit activity), ruling out the possibility that delta spikes merely reflect the overall level of hippocampal excitatory drive during ripples. Finally, ripples facilitated (but did not entirely control) the formation of delta assemblies (Fig. S7). Further, the same GLM analysis applied to hippocampal and cortical ensembles showed that hippocampal activity could even predict delta components (Fig. 2D).

Our second prediction concerned the relation of this predictive bias to behavior. In sleep sessions preceding the task, hippocampal spiking activity during ripples failed to predict subsequent delta spikes or assemblies (Fig. S10), indicating that the predictive bias emerged following task performance. We then investigated the behavioral correlates of the prefrontal units whose delta spikes were significantly predicted by hippocampal ripple activity during sleep after behavior ('partner cells', Fig. S11; see Tables S1 and S2). These cortical cells displayed higher levels of task-relevant firing during behavior (Fig. 2E; we failed to find a similar effect for delta components, possibly because of low statistical power due to their limited number: $n=14$ components, $n=9$ predicted). We further investigated the behavioral correlates of delta assemblies, and found that these assemblies were also expressed during task performance, but not outside delta waves nor in sleep preceding behavior (Fig. S12).

These observations suggest that in addition to triggering the reorganization of cortical subnetworks during the transition to the up state (5), an unsuspected role of the delta wave may be to isolate from interference specific cortical computations taking place in response to hippocampal replay. Consistent with this idea, delta waves typically occurred within a critical time window where cortical ac- tivity remained correlated with hippocampal ripple activity (Fig. 3A). To test if delta waves tended to preferentially silence cortical activity that was unrelated to the ongoing hippocampo-cortical dialogue, we classified individual prefrontal spikes as 'partner spikes' if they followed spikes emitted by their significantly correlated hippocampal units, or 'other spikes' if they were unrelated to the preceding hippocampal activity (Fig. 3B; more examples are shown in Fig. S13). The signal-to-noise ratio for partner spikes peaked during delta waves (Fig. 3C), and this was due to the selective silencing of nonpartner activity during delta waves (Fig. S14).

Does this isolation of cortical computations play a critical role in memory consolidation? A prediction of this hypothesis is that isolating cortical assemblies by experimental induction of delta waves should trigger memory consolidation, but only if the isolated activity is relevant to the hippocampocortical dialogue (partner spikes). We have already shown that triggering delta waves when endogenous mechanisms fail to do so, can boost memory consolidation provided the delta waves are induced in an appropriate time window (Fig. 4A, (5)). We thus sought to confirm the prediction that these delta waves actually isolated partner spikes, i.e. that delta spikes did occur during induced delta waves, and that they were predicted by hippocampal activity. Similar to our observations in natural sleep (above), stimulation-induced delta waves did feature spiking activity (Fig. S15), and these delta spikes were predicted by preceding hippocampal activity coinciding with the timing of ripples (Fig. 4BD). In contrast, slightly delaying the induction of delta waves (by $\sim 200 \mathrm{~ms}$, see Fig. 3A) to isolate non-partner delta spikes (Fig. 4B-D) failed to induce memory consolidation (5).

Our results challenge the generally accepted tenet that delta waves, reflecting the down states of the sleep slow oscillation, are periods of complete cortical silence $(1,6,25)$ - to the point that they have sometimes been defined as such (26, 27), and that occasional spikes have been routinely ignored when detected $(28,29)$. We focused on delta spikes and found that they are not neuronal noise due to imperfect silencing of the cortical mantle. On the contrary, they constitute a common phenomenon potentially implicating all neurons and all delta waves, and they reflect genuine processing involved in memory consolidation.

This also provides a mechanism for the documented but puzzling role of delta waves in memory consolidation: synchronized silence across most 
A
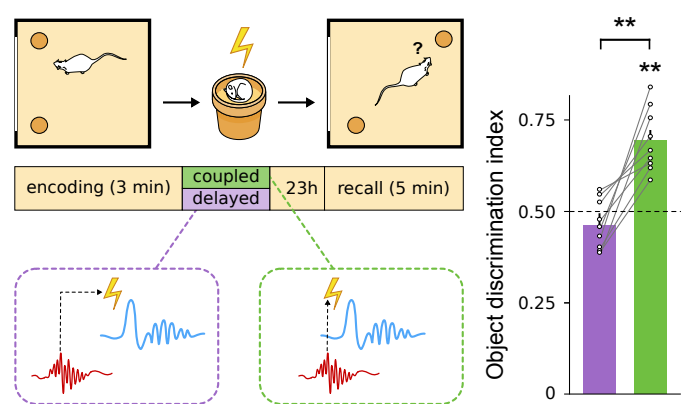

C

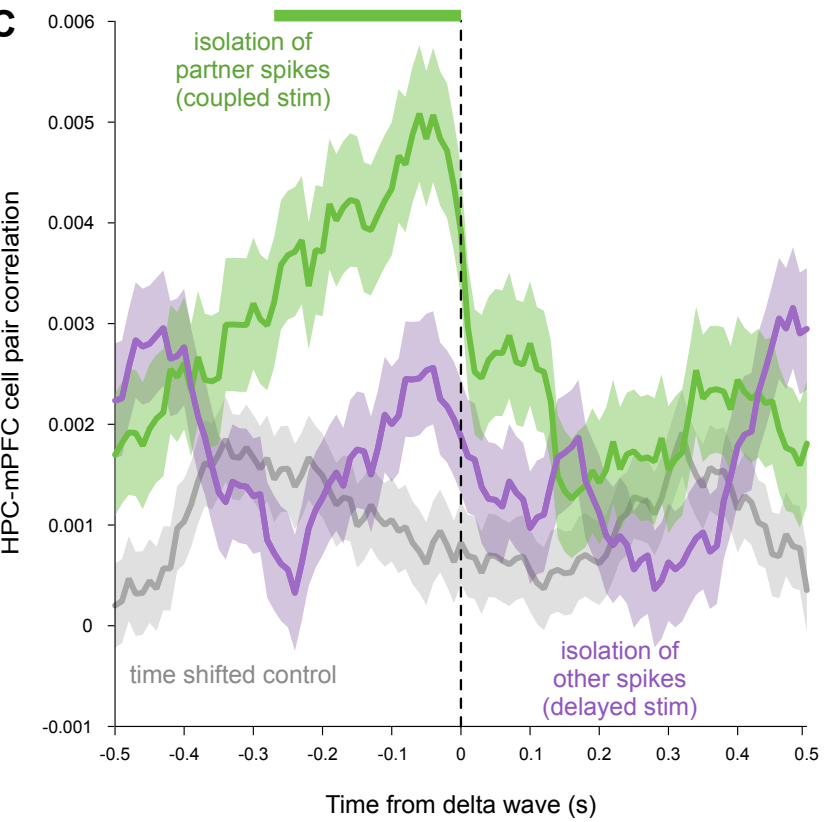

B
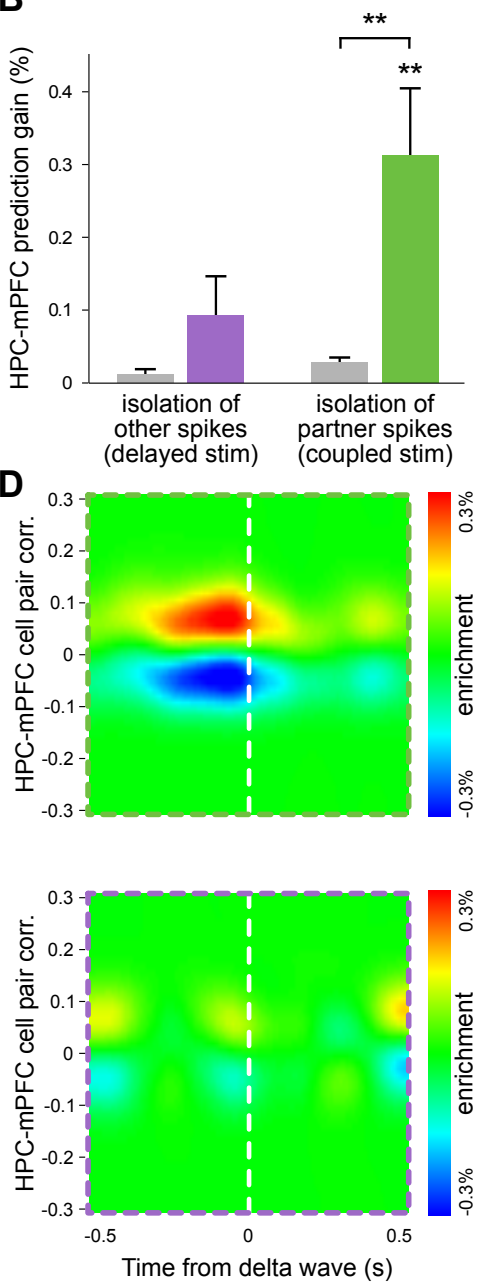

Fig. 4. Induction of memory consolidation by isolation of partner spikes. (A) Left: experimental protocol. Delta waves were induced by brief single-pulse electrical stimulation of deep cortical layers. Induced delta waves were triggered to isolate either partner activity (coupled stimulation; green, $130 \mathrm{~ms}$ after ripples) or other cortical activity (delayed stimulation; purple, 290-370 ms after ripples) during sleep following limited training on a spatial object recognition task. Right: Object discrimination index during the recall phase. Only delta waves triggered to isolate partner activity (coupled stimulation) resulted in memory consolidation and enhanced task performance. (B) Performance of a GLM trained to predict delta spikes based on preceding hippocampal activity (200 ms window), measured as percent improvement relative to a shuffled control (prediction gain). Only delta waves triggered to isolate partner activity did result in significant prediction of delta spikes $(P=0.0030$ for isolation of partner spikes by coupled stimulation, $P=0.1301$ for isolation of other spikes by delayed stimulation, Wilcoxon rank-sum test). (C) Cross-correlation (curves and shaded areas, mean \pm s.e.m.) of hippocampal activity and delta spikes (green: isolation of partner spikes by coupled stimulation; purple: isolation of other spikes by delayed stimulation; grey: time-shifted control; horizontal green line: Monte-Carlo test, $P<0.05)$. (D) Enrichment in positive correlations upon isolation of partner spikes by coupled stimulation (top) but not of other spikes by delayed stimulation (bottom).

of the cortex isolates the network from competing inputs, while a select subpopulation of neurons maintains relevant spike patterns active between epochs of hippocampo-cortical information transfer $(10,12,14)$, and epochs of cortical plasticity $(15,16)$ and network reorganization $(5,18,19)$. Yet, in many cases cortical activity during delta waves could not be reliably predicted from the preceding hippocam- pal ripple activity. It is conceivable that such cortical activity could instead have been related to interactions with other brain networks. This suggests that delta spikes and assemblies might constitute a general mechanism of isolated cortical computation beyond the hippocampo-cortical dialogue. 
Acknowledgments We thank Nicolas Maingret for collecting the data and Romain Fayat for help with the delta wave detection algorithm. Funding. This work was supported by the Agence Nationale de la Recherche (ANR15-CE16-0001-02, ANR-17-CE37-0016-01), Collège de France (R.T.), and a joint grant from École des Neurosciences de Paris Île-de-France and LabEx MemoLife (ANR-10-LABX54 MEMO LIFE, ANR-10-IDEX-0001-02 PSL*) (R.T.) Author Contributions. M.Z. designed the study. R.T. and M.Z. designed the analyses. R.T. performed the analyses. R.T. and M.Z. wrote the manuscript. Competing Interests. The authors declare no competing interests. Data and Materials Availability. Data of this study are available at https://doi.org/10.6080/K0TD9VJG (30). This is the author's version of the work. It is posted here by permission of the AAAS for personal use, not for redistribution. The definitive version was published here.

\section{References}

1. M. Steriade, A. Nunez, F. Amzica, Journal of Neuroscience 13, 3252 (1993).

2. H.-V. V. Ngo, T. Martinetz, J. Born, M. Mölle, Neuron 78, 545 (2013).

3. L. Marshall, H. Helgadottir, M. Molle, J. Born, Nature 444, 610 (2006).

4. S. Chauvette, J. Seigneur, I. Timofeev, Neuron 75, 1105 (2012).

5. N. Maingret, G. Girardeau, R. Todorova, M. Goutierre, M. Zugaro, Nature Neuroscience 19, 959 (2016).

6. A. Sirota, G. Buzsáki, Thalamus and Related Systems 3, 245 (2005)

7. A. Sirota, J. Csicsvari, D. Buhl, G. Buzsáki, Proceedings of the National Academy of Sciences 100, 2065 (2003).

8. G. Girardeau, K. Benchenane, S. I. Wiener, G. Buzsáki, M. B. Zugaro, Nature Neuroscience 12, 1222 (2009).

9. V. Ego-Stengel, M. A. Wilson, Hippocampus 20, 1 (2010).

10. A. K. Lee, M. A. Wilson, Neuron 36, 1183 (2002).

11. D. Ji, M. A. Wilson, Nature neuroscience 10, 100 (2007).
12. G. Rothschild, E. Eban, L. M. Frank, Nature Neuroscience 20, 251 (2017).

13. C. M. Wierzynski, E. V. Lubenov, M. Gu, A. G. Siapas, Neuron 61, 587 (2009).

14. A. Peyrache, M. Khamassi, K. Benchenane, S. I. Wiener, F. P. Battaglia, Nature Neuroscience 12, 919 (2009).

15. P. B. Kruskal, L. Li, J. N. MacLean, Nature Communications 4, 2574 (2013).

16. T. Gulati, L. Guo, D. S. Ramanathan, A. Bodepudi, K. Ganguly, Nature Neuroscience 20, 1277 (2017).

17. A. G. Siapas, M. A. Wilson, Neuron 21, 1123 (1998).

18. M. Rosanova, D. Ulrich, The Journal of Neuroscience 25, 9398 (2005).

19. J. Seibt, et al., Nature Communications 8, 684 (2017).

20. D. Marr, Philosophical Transactions of the Royal Society B: Biological Sciences 262, 23 (1971).

21. G. Buzsáki, Neuroscience 31, 551 (1989).

22. P. W. Frankland, B. Bontempi, Nature Reviews Neuroscience 6, 119 (2005).

23. V. L. dos Santos, S. Ribeiro, A. B. L. Tort, J. Neurosci. Methods 220, 149 (2013).

24. K. D. Harris, J. Csicsvari, H. Hirase, G. Dragoi, G. Buzsáki, Nature 424, 552 (2003).

25. M. Steriade, I. Timofeev, Neuron 37, 563 (2003).

26. A. Luczak, P. Barthó, S. L. Marguet, G. Buzsáki, K. D. Harris, Proceedings of the National Academy of Sciences 104, 347 (2007).

27. L. Johnson, D. Euston, M. Tatsuno, B. McNaughton, The Journal of neuroscience 30, 2650 (2010).

28. J. Csicsvari, et al., Journal of Neurophysiology 90, 1314 (2003).

29. D. Jercog, et al., eLife 6, e22425 (2017).

30. N. Maingret, R. Todorova, M. Zugaro (2019).

31. L. Hazan, M. Zugaro, G. Buzsáki, J Neurosci Methods pp. 207-216 (2006).

32. P. Barthó, et al., J Neurophysiol 92, 600 (2004).

33. G. M. van de Ven, S. Trouche, C. G. McNamara, K. Allen, D. Dupret, Neuron 92, 968 (2016).

34. J. Willing, J. M. Juraska, Neuroscience 301, 268 (2015). 


\section{Materials and Methods}

\section{Animals}

The analyses presented here were performed on data collected for a previous study by Maingret et al. (5). Briefly, a total of 9 male Long Evans rats (René Janvier, Le Genest, St Isle, France; weight, 280-350 g) were maintained on a 12h:12h light-dark cycle (lights on at $7 \mathrm{am})$. Training and experiments took place during the day. Rats were grouphoused until one week before surgery. All experiments were in accord with institutional (CNRS Comité Opérationnel pour l'Éthique dans les Sciences de la Vie) and international (US National Institutes of Health guidelines) standards, legal regulations (Certificat no. B751756), and ethical requirements (Ethics Committee approval \#2012-0048) regarding the use and care of animals.

\section{Surgery}

Rats $(n=9)$ were deeply anesthetized (xylazine, $0.1 \mathrm{ml}$ intramuscular; pentobarbital, $40 \mathrm{mg}$ per $\mathrm{kg}$ of body weight, intraperitoneal; $0.1 \mathrm{ml}$ pentobarbital supplemented every hour) and implanted with a custom-built microdrive with 16 individual tetrodes (groups of four twisted $12 \mu \mathrm{m}$ tungsten wires, gold-plated to $\sim 200 \mathrm{k} \Omega$ ), of which 8 targeted the prelimbic and infralimbic regions of the right $\mathrm{mPFC}$ (AP: $+2.7 \mathrm{~mm}$ from bregma; ML: $+1.5 \mathrm{~mm}$, angled at $10^{\circ}$ from the sagittal plane), and the other 8 targeted the CA1 subfield of the right hippocampus (AP: -3.5 to $-5.5 \mathrm{~mm}$; ML: +2.5 to $+5.0 \mathrm{~mm}$ ). A custombuilt bipolar stimulation electrode consisting of two stainless steel wires (total length, $1.5 \mathrm{~mm}$; inter-wire interval, $0.5 \mathrm{~mm}$; wire diameter, $70 \mu \mathrm{m})$ was implanted in the left neocortex (AP: $+2 \mathrm{~mm}$; ML: $-2 \mathrm{~mm}$; DV: $-1.5 \mathrm{~mm}$ from the dura, motor area). Miniature stainless steel screws (reference and ground) were implanted above the cerebellum. During recovery from surgery (minimum 3 days), the rats received food and water ad libitum. The recording electrodes were then progressively lowered until they reached their targets and then adjusted every day to optimize yield and stability. 


\section{Behavioral task}

The spatial object recognition task has been described previously (5). Briefly, during a 3 min encoding phase, the rats explored a $70 \mathrm{~cm} \times 50 \mathrm{~cm}$ arena with two identical objects placed in two adjacent corners. The rats were then placed in a flower pot for sleep sessions, which lasted until 1,000 stimulations had been delivered (see below; 4,000 s of SWS), then returned to their home cage. On the following day, during a 5 min recall phase, the rats explored the same arena, in which one of the objects had been displaced to the opposite corner. The rats were then placed in a flower pot for uninterrupted sleep sessions without stimulation. After an interval of at least two days, each rat performed the task a second time with different objects. The order of the stimulation conditions was pseudo-randomly distributed among rats.

\section{Stimulation protocol}

The stimulation protocol has been described previously (5). Briefly, threshold crossing on the ripple band-filtered hippocampal signal automatically triggered a monophasic singlepulse $(0.1 \mathrm{~ms})$ stimulation of the deep layers of the motor cortex, delivered by a constant current stimulator (SD9 square pulse stimulator, Grass Technologies). For each animal, the minimum voltage necessary to reliably induce propagating delta waves was determined prior to training (range: 17.5-22.5 V). The number of stimulations was limited to one every two seconds, and the total number of stimulations was set to 1,000, yielding a stimulation period of $\sim 4,000 \mathrm{~s}$. To isolate partner cells, stimulations were delivered to trigger a delta wave $\sim 130$ ms following SPW-R detection, emulating endogenous fine-tuned SPWR-delta coordination. To isolate other (non-partner) cells, an additional random delay (range: 160-240 ms) was introduced between SPW-R detection and stimulation onset.

\section{Data acquisition and processing}

Brain signals were preamplified (unity-gain headstages, Noted Bt, Pécs, Hungary), amplified $\times 500$ (Neuralynx L8, Bozeman, MT, USA), acquired and digitized with two synchronized Power1401 systems (CED, Cambridge, UK). A red LED was used to track the 
instantaneous position of the animals $(25 \mathrm{~Hz})$. For off-line spike sorting, the wide-band signals were converted, digitally high-pass filtered (nonlinear median-based filter) and thresholded, and waveforms were extracted and projected to a PCA subspace using NDManager (L. Hazan and M. Zugaro, http://neurosuite.sourceforge.net, (31)). Spike sorting used a semi-automatic cluster cutting procedure combining KlustaKwik (K.D. Harris, http://klustakwik.sourceforge.net) and Klusters (L. Hazan, http://neurosuite. sourceforge.net, (31)). In the prefrontal cortex, excitatory and inhibitory cells were discriminated based on significant short-latency peaks and troughs in cross-correlograms (32), that were further validated by visual inspection. In addition, units were divided into putative pyramidal cells and interneurons based on half-amplitude duration and trough to peak time (32). In the hippocampus, putative pyramidal cells were identified based on firing rate and bursting properties. Neurophysiological and behavioral data were explored using NeuroScope (L. Hazan, http://neurosuite.sourceforge.net, (31)). LFPs were derived from wideband signals by downsampling all channels to 1,250 Hz.

At the end of the experiments, recording sites were marked with small electrolytic lesions. Rats were deeply anesthetized with a lethal dose of pentobarbital, and intracardially perfused with saline $(0.9 \%)$ followed by paraformaldehyde $(10 \%)$. Coronal slices (40 $\mathrm{\mu m})$ were stained with cresyl-violet.

\section{Data analysis and statistics}

Data were analyzed using in Matlab (MathWorks, Natick, MA), using FMAToolbox (http://fmatoolbox.sourceforge.net) and custom written programs. Spectrograms were constructed using Chronux (http://chronux.org/).

\section{Object responsivity}

Object responsivity was computed as described previously (5). Briefly, the responsivity index $R$ was defined as the mean firing rate $r$ over the quadrants containing the objects, and z-scored relative to the distribution $F$ of firing rates in the empty quadrants, i.e. $R=(r-\mu) / \sigma$ where $\mu$ and $\sigma$ are the mean and standard deviation of $F$. Thus, the 
object responsivity index $R$ measured by how much, relative to its baseline variability, a cell increased its firing rate around the objects.

\section{Sleep scoring}

Sleep stages (SWS vs REM) were determined by automatic K-means clustering of the theta/delta ratio extracted from the power spectrograms during the episodes where the animal was immobile (linear velocity $<3 \mathrm{~cm} / \mathrm{s}$ for at least $30 \mathrm{~s}$, with brief movements $<0.5 \mathrm{~s})$.

\section{Ripple detection}

To detect ripple events, we first detrended the LFP signals and used the Hilbert transform to compute the ripple band $(100-250 \mathrm{~Hz})$ amplitude for each channel recorded from the CA1 pyramidal layer. We then averaged these amplitudes, yielding the mean instantaneous ripple amplitude. To exclude events of high spectral power not specific to the ripple band, we then subtracted the mean high-frequency $(300-500 \mathrm{~Hz})$ amplitude (if the difference was negative, we set it to 0). Finally, we z-scored this signal, yielding a corrected and normalized ripple amplitude $R(t)$. Ripples were defined as events where $R(t)$ crossed a threshold of 3 s.d. and remained above 1 s.d. for 30 to $110 \mathrm{~ms}$.

\section{Delta wave detection}

Delta waves were identified based on detection of both large positive deflections in the LFP and concurrent decreases in multiunit activity. First, the LFP recorded from each tetrode located in the mPFC was filtered $(0-6 \mathrm{~Hz})$ and z-scored, yielding $D(t)$. The beginning $\left(t_{\text {beginning }}\right)$, peak $\left(t_{\text {peak }}\right)$, and end $\left(t_{\text {end }}\right)$ of putative delta waves were defined as upward-downward-upward zero-crossings of $D^{\prime}(t)$. Epochs where either $D\left(t_{\text {peak }}\right)>2$ and $D\left(t_{\text {end }}\right)<0$, or $D\left(t_{\text {peak }}\right)>1$ and $D\left(t_{\text {end }}\right)<-1.5$, were deemed candidate events. Candidate events briefer than $150 \mathrm{~ms}$ or longer than $500 \mathrm{~ms}$ were discarded. Second, the instantaneous mPFC multiunit activity was smoothed (Gaussian window, $\sigma=60 \mathrm{~ms}$ ). Candidate events where the smoothed activity decreased relative to a $2 \mathrm{~s}$ period around $t_{\text {peak }}$ were considered delta waves. 


\section{Delta spikes and assemblies}

To quantify spiking during delta waves, we computed the distribution of delays between a delta wave peak and the nearest spike emitted by each neuron recorded on the same tetrode as the delta wave. This yielded a bimodal distribution (Fig. 1C): while most nearest spikes occurred 100-200 ms from the delta wave peak (i.e. in the UP state), in a minority of cases a spike occurred within $15 \mathrm{~ms}$ of the delta wave peak. Using this value as a conservative threshold, we defined delta spikes as spikes emitted within $15 \mathrm{~ms}$ of the peak of a delta wave detected on the same tetrode as the active neuron (all analyses were repeated and confirmed using $\pm 30 \mathrm{~ms}$ time windows). Delta waves in which we did not detect any delta spikes were referred to as silent delta waves.

We used independent component analysis (ICA) to detect significant cell assemblies (33). We computed a delta spike matrix $M$, where $M(i, j)$ is the number of spikes emitted by neuron $i$ within $15 \mathrm{~ms}$ of delta wave $j$. We z-scored $M$ and projected it onto its $n$ significant principal components - the $n$ components with eigenvalues exceeding the Marcenko-Pastur threshold $\lambda_{\max }$. We then ran ICA (using the FastICA package for Matlab, http://research.ics.aalto.fi/ica/fastica) on the projected matrix to obtain the weight vectors describing the assemblies. The activation of a given cell assembly $k$ was computed as $A_{k}=M^{T} P_{k} M$, where $P_{k}$ is the projection operator of the cell assembly (the outer product of its weight vector) with its diagonal set to zero.

To assess whether the detected activity patterns were also expressed in other behavioral conditions or brain states (Fig. S12), we detected cell assemblies separately in five different conditions: delta waves in pre-task and post-task sleep, non-delta periods in pre-task and post-task sleep (100 ms non-overlapping bins excluding delta waves), and task-related behavior (100 ms non-overlapping bins). To estimate similarity across the patterns in any two conditions, each component in one condition was matched to the component in the other condition which yielded the highest absolute correlation, and the average correlation between matched components was computed (adapted from van de Ven et al. (33)). To assess significance, we compared this average correlation to a distribution of 
shuffled correlations obtained by permuting the weights of each component across cells and reiterating the above procedure (matching and computing average correlation between matched components) 1,000 times.

Cooperative activity within delta waves was assessed by quantifying to what extent the delta spike activity of each prefrontal cortical cell could be predicted from the delta spike activity of all other prefrontal cortical cells ('peer prediction' (24)). Thus, for each prefrontal cortical neuron $i$, delta waves were split into $n_{i}$ non-overlapping partitions, ensuring that each partition contained exactly one delta wave where neuron $i$ fired. Using a subset of $\left(n_{i}-1\right)$ partitions, a Generalized Linear Model (GLM) was trained to predict the binary delta spike vector $D_{i}$ of neuron $i$, from the matrix $D_{(i)}$ containing the delta spike counts of all other prefrontal neurons (i.e. excluding neuron $i$ ). The GLM was tested on the remaining partition. This procedure was repeated $n_{i}$ times, each time using a different subset of training and test partitions, resulting in multi-fold cross-validation. Following (12), the quality of the prediction was assessed by comparing the median prediction error $e$ to the median error $e_{\text {shuffled }}$ obtained by shuffling 1,000 times the predictions relative to the observed data $D_{i}$. The prediction gain $g$ was defined as $g=e_{\text {shuffled }} / e$.

\section{Prediction of delta spikes by hippocampal activity}

Cross-correlations. Cross-correlations between hippocampal ripple activity and surrounding prefrontal cortical activity (shown in Fig. 3A) were computed as follows. For each hippocampal pyramidal unit $i$, spikes were counted in $200 \mathrm{~ms}$ windows centered on each $t_{\text {ripple }}$, yielding a spike count vector $N_{i}$. Similarly, for each prefrontal cortical unit $j$, spikes were counted in $200 \mathrm{~ms}$ windows centered on $t_{\text {ripple }}+k(k \in[-500,500] \mathrm{ms})$, yielding a spike count vector $N_{j}(k)$ for each temporal shift $k$. The Spearman rank-order correlation $\rho_{i j}(k)$ between $N_{i}$ and $N_{j}(k)$ was averaged over $i$ and $j$, yielding the mean cross-correlation $\rho(k)$ as a function of the temporal shift $k$.

Cross-correlations between delta spikes and surrounding hippocampal ripple activity (shown in Fig. 2B) were computed using a similar procedure, where delta spikes were counted in $30 \mathrm{~ms}$ windows centered on $t_{\text {delta }}$ (corresponding to the $15 \mathrm{~ms}$ threshold dis- 
tance defined in Fig. 1C), and hippocampal ripple spikes were counted in $200 \mathrm{~ms}$ windows centered on $t_{\text {delta }}+k$. Two additional criteria were applied for inclusion in this analysis. Firstly, for a given prefrontal cortical unit $i$, only delta waves recorded from the same tetrode were considered. Secondly, for a given value of $k$, delta waves were taken into account only when ripple activity took place within a $200 \mathrm{~ms}$ window centered on $t_{\text {delta }}+k$. Prefrontal cortical cells that participated in at least one significant cross-structural cell pair for $k=-200 \mathrm{~ms}$ were defined as partner cells.

Cross-correlations between hippocampal spikes and delta assemblies were computed using the same procedure, but using assembly activations $A_{i}$ (see section Delta spikes and assemblies, above), rather than spike counts, during delta waves.

To assess the significance of the cross-correlations described above, we generated control cross-correlations $\rho_{i j}^{\text {shifted }}(k)$ by shifting one of the temporal series: for instance, hippocampal activity surrounding the $n$-th delta wave was correlated with delta spikes emitted during the $(n+1)$-th delta wave, thus shifting the correlation by one delta wave. This approach, as opposed to a completely random shuffle of all events, selectively disrupted fine timescale correlations but preserved long timescale trends, effectively preventing false positives due to non-specific factors such as sleep progression or depth.

When these analyses were repeated after removing from the spike trains all hippocampal spikes emitted outside ripples, all results were confirmed.

Enrichment (Figs. 2C and 4D) was defined as the difference between observed and spurious correlations $\rho_{i j}(k)-\rho_{i j}^{\text {shifted }}(k)$.

Generalized Linear Model (GLM). The predictive power of hippocampal ripple activity on prefrontal delta spikes was further assessed using a GLM analysis, which considered ensemble activity rather than neuron pairs (as in the cross-correlation analyses above). The procedure was similar to the peer prediction method described for prefrontal cooperative activity during delta waves. Briefly, a GLM was trained to predict the binary delta spike vector $D_{i}$ of prefrontal cortical neuron $i$, from the matrix $H$ containing the spike counts of hippocampal pyramidal neurons in $200 \mathrm{~ms}$ windows preceding delta waves 
( $H_{m j}$ is the number of spikes emitted by neuron $j$ before the $m$-th delta wave). The same multi-fold cross-validation scheme was used as above, yielding a prediction gain $g=e_{\text {shuffled }} / e$. When these analyses were repeated after removing from the spike trains all hippocampal spikes emitted outside ripples, all results were confirmed.

Predicted spikes and signal-to-noise ratio. This analysis examined hippocampocortical correlations around each delta wave $k$ (not restricted to delta spikes). As in the previous cross-correlation analyses, partner cells were first identified based on significant Spearman's rank correlation (here, between prefrontal $N_{i}(k)$ and hippocampal $\left.N_{j}(k-200 \mathrm{~ms})\right)$. Subsequently, for each prefrontal cortical unit, individual spikes were deemed 'partner spikes' if any of the unit's partner cells in the hippocampus fired within a preceding $200 \mathrm{~ms}$ window. The signal-to-noise ratio measures the ratio of partner spikes versus other spikes in $20 \mathrm{~ms}$ bins around delta waves. 
A
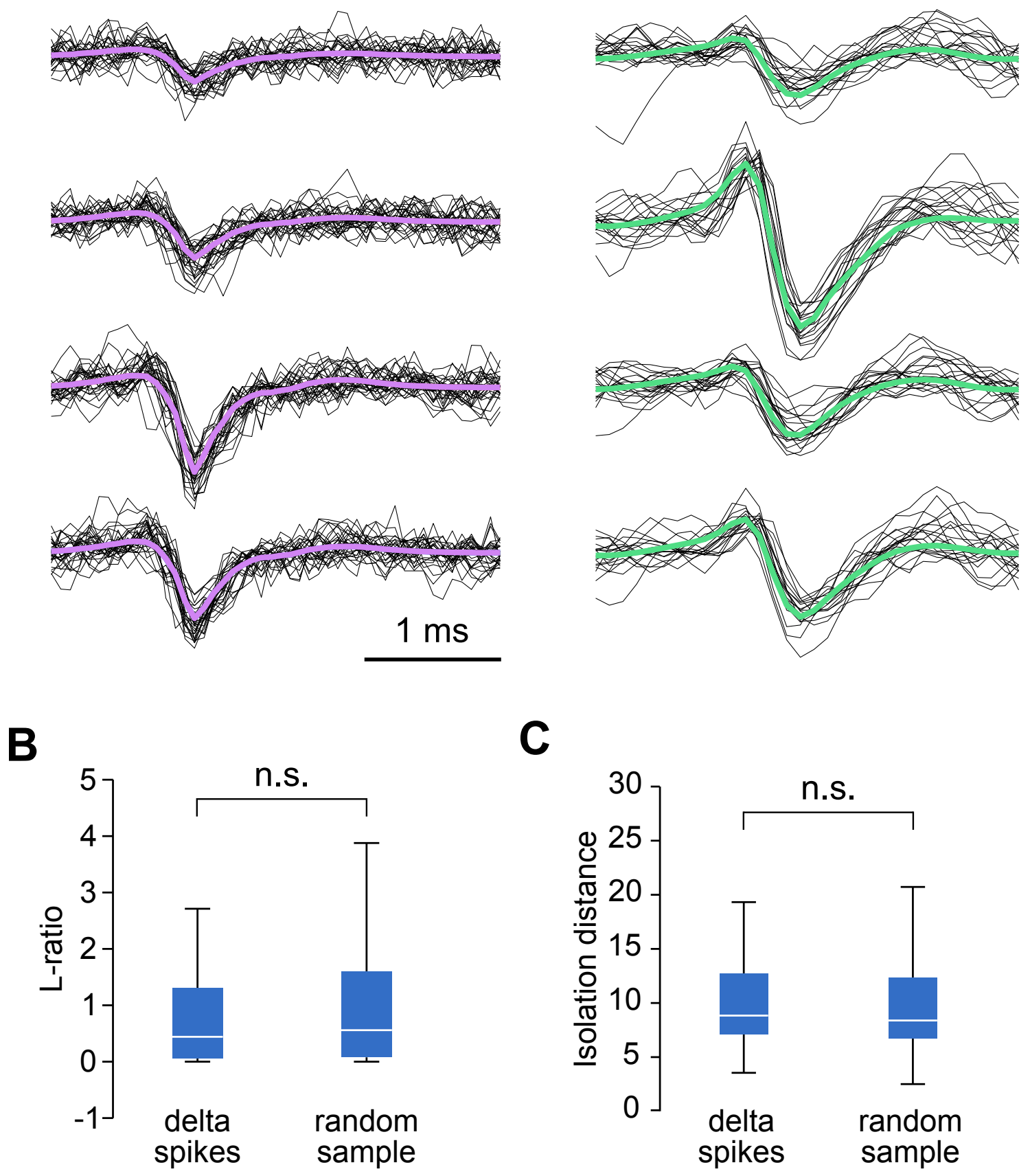

C

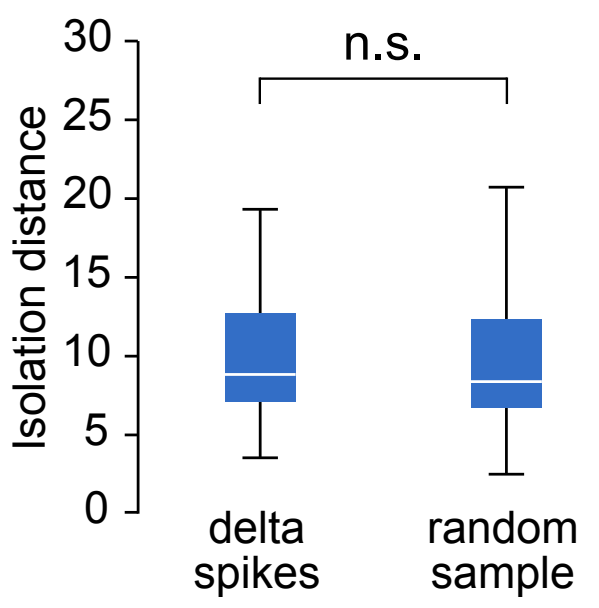

Fig. S1. Delta spike waveforms. (A) Spike waveforms for all the recorded delta spikes (black traces) for two example units. Colored traces: average waveforms for all spikes that the same units fired outside delta waves. (B) L-ratio was not different $(P=0.72279$, Wilcoxon rank-sum test) between delta spikes and a random sample of non-delta spikes. (C) Isolation distance was not different ( $P=0.84504$, Wilcoxon rank-sum test) between delta spikes and a random sample of non-delta spikes. 
A

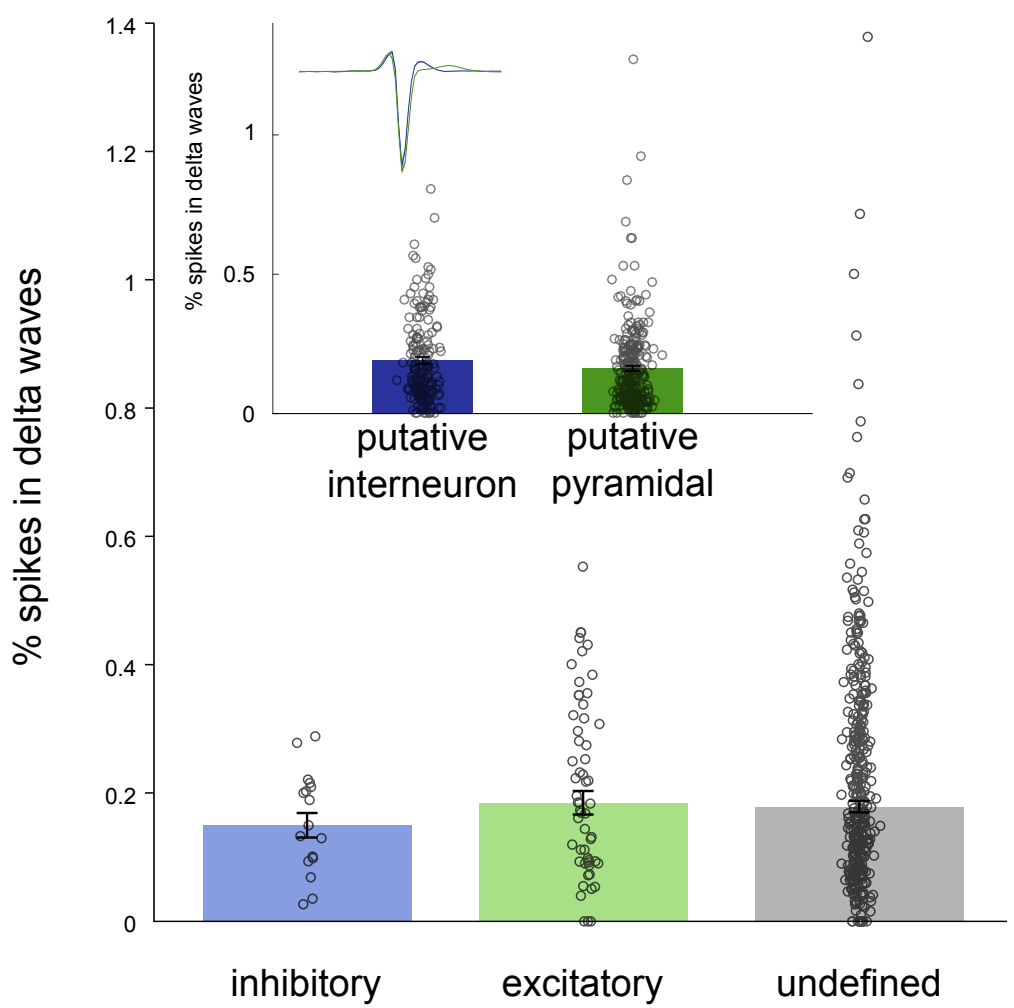

B
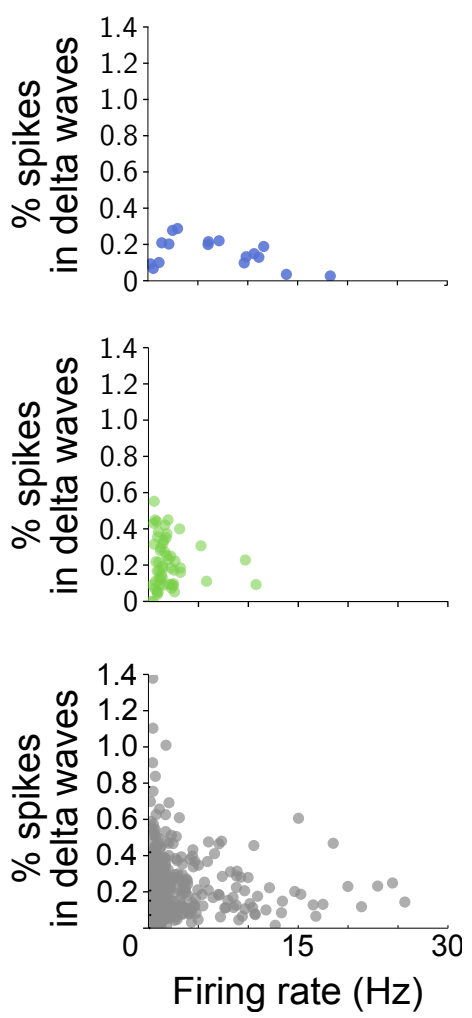

Fig. S2. Delta wave firing across units. (A) During SWS, the proportion of spikes fired in delta waves did not differ between CCG-identified inhibitory $(n=17)$, excitatory $(n=57)$ and undefined $(n=366)$ cells $(P=0.5322$, Kruskal-Wallis test). Inset: units were additionally divided according to waveform. The proportion of spikes fired in delta waves did not differ between waveform-identified putative interneurons and putative pyramidal cells $(P=0.0734$, Wilcoxon rank-sum test). (B) There was no correlation $(P>0.05)$ between firing rate and the proportion of SWS spikes fired in delta waves for any of the cell types (top, excitatory cells; middle, inhibitory cells; bottom: undefined cells). 
A
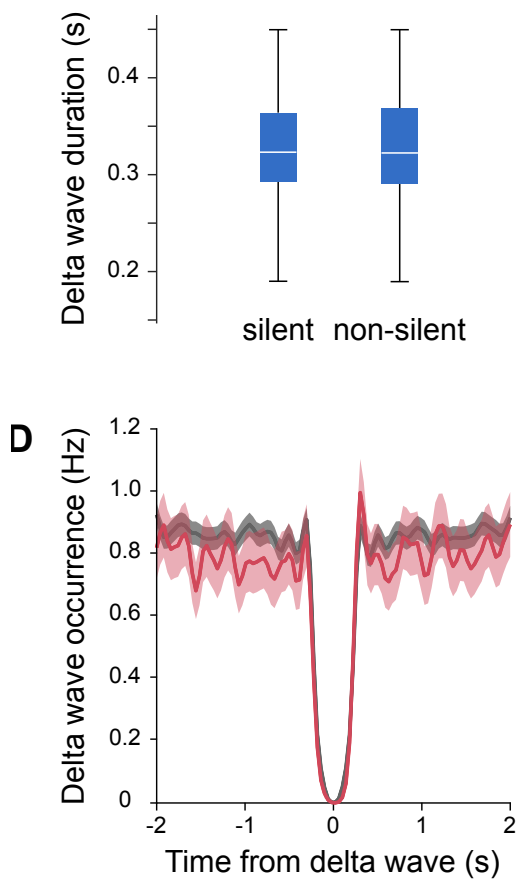

B
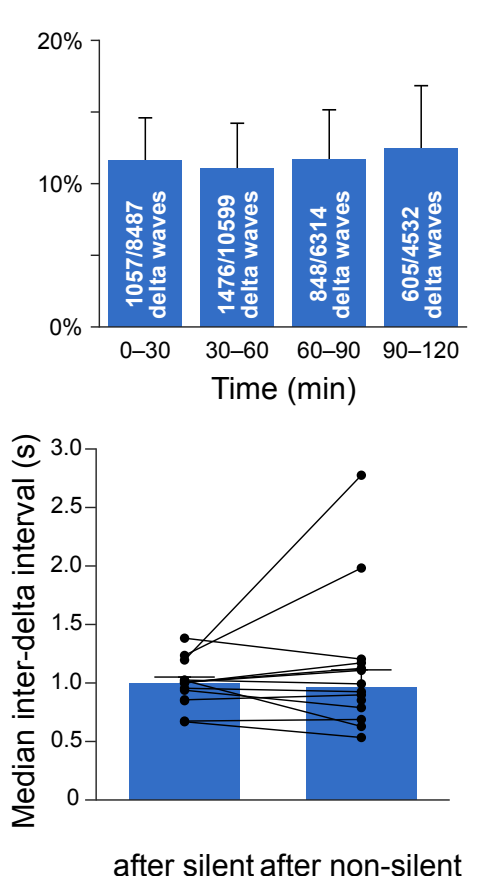

C
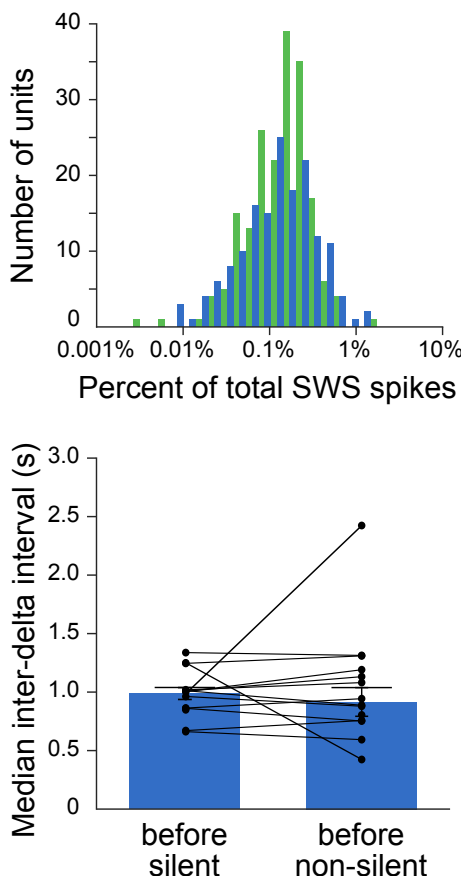

Fig. S3. Timing of silent and non-silent delta waves. (A) Delta wave duration was not different between silent $(n=21,733)$ and non-silent $(n=7,162)$ delta waves $(P=0.45582$, Wilcoxon rank-sum test). (B) The proportion of non-silent delta waves did not vary across sleep ( $P>0.05$, binomial proportion tests). (C) The proportion of SWS spikes fired in delta waves for each unit was not different between pre-task (blue) and post-task (green) sleep ( $P=0.78105$, Wilcoxon signed rank test). (D) Delta wave timing with respect to other delta waves was not different between silent and non-silent delta waves. Left: delta-wave occurrence rate centered on silent (black) and non-silent (red) delta waves (curves and shaded area, mean \pm s.e.m.) Note that silent delta waves were not more likely to be preceded or followed by other delta waves than silent delta waves. Center: median inter-delta intervals were not significantly different following silent vs non-silent delta waves $(P=0.76086$, Wilcoxon signed rank test; bars: median \pm s.e.median; dots: within-session medians). Right: median inter-delta intervals were not significantly different preceding silent vs non-silent delta waves $(P=0.80774$, Wilcoxon signed rank test; bars: median \pm s.e.median; dots: within-session medians). 
$\mathbf{A}$
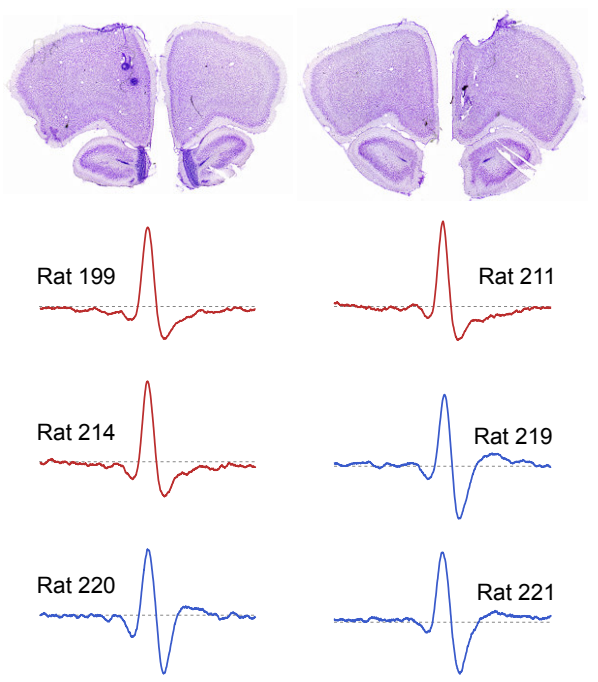

D
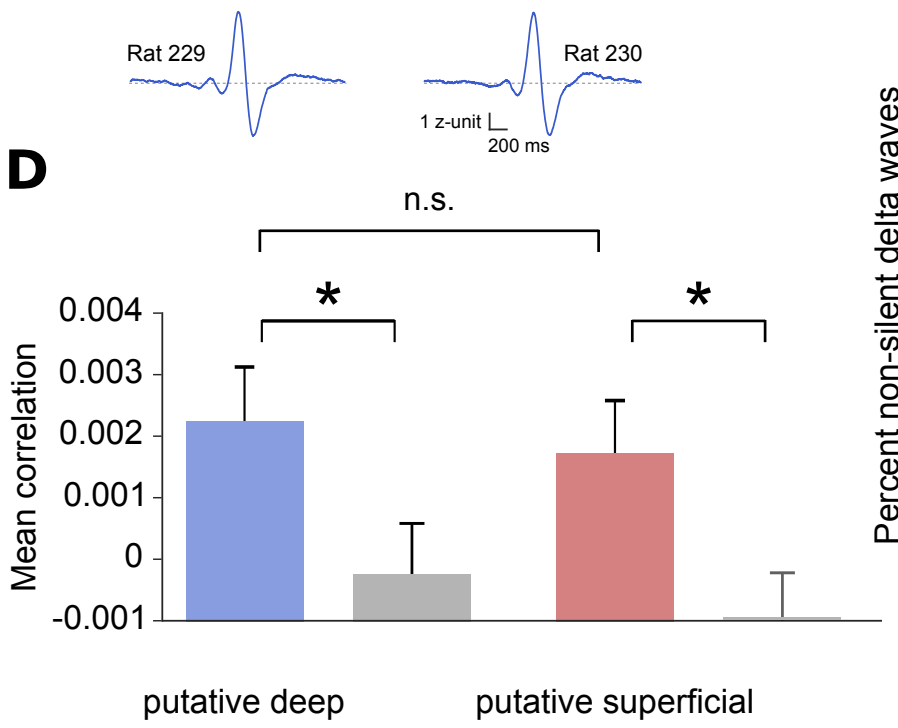

B

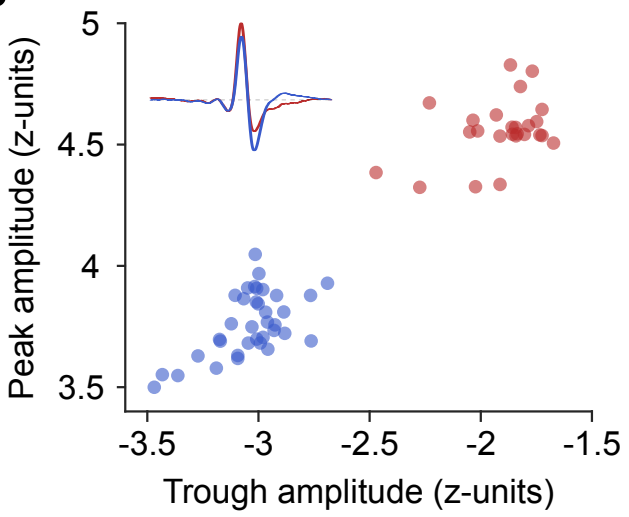

C

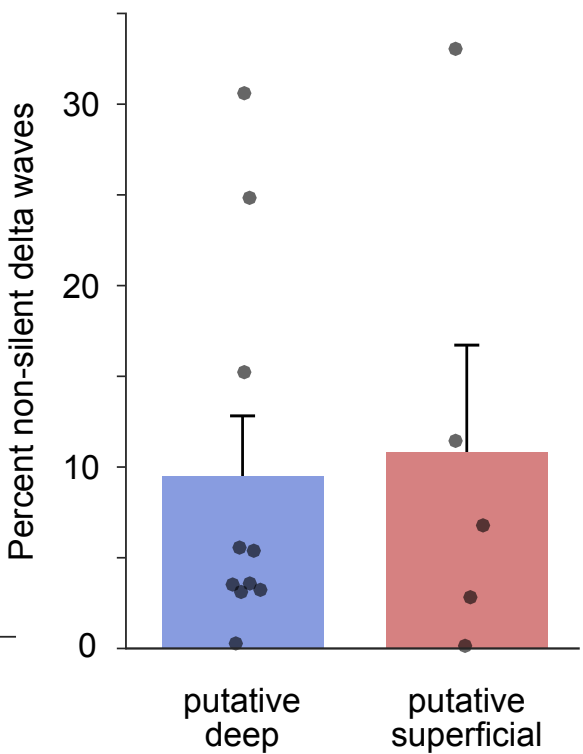

Fig. S4. Delta spikes recorded from different cortical layers. (A) Top: example histological sections showing electrode penetrations at various depths. Bottom: average waveforms for delta waves recorded from example electrodes in each rat (red: putative more superficial recording sites, blue: putative deeper recording sites). (B) Scatterplot of average delta wave trough and peak amplitudes for each recorded electrode group. (C) The proportion of nonsilent delta waves was not significantly different between recordings from putative deep (blue) and recordings from putative superficial (red) recording sites (dots: individual recording sessions; bars: mean \pm s.e.m, $P=0.95305$, Wilcoxon rank-sum test). (D) Hippocampal ripple activity predicts delta spikes independently of the depth of the recording site. Correlations between hippocampal ripple activity (recorded in $200 \mathrm{~ms}$ windows preceding delta waves) and delta spikes were significantly greater than in time-shifted controls (grey bars) for recordings in both putative deep (blue, $P=0.028$, Monte-Carlo test) and superficial (red, $P=0.028$, Monte-Carlo test) sites. There was no significant difference between more superficial and deeper recording sites ( $P=0.674$, Monte-Carlo test) (bars: mean \pm s.e.m). 
A

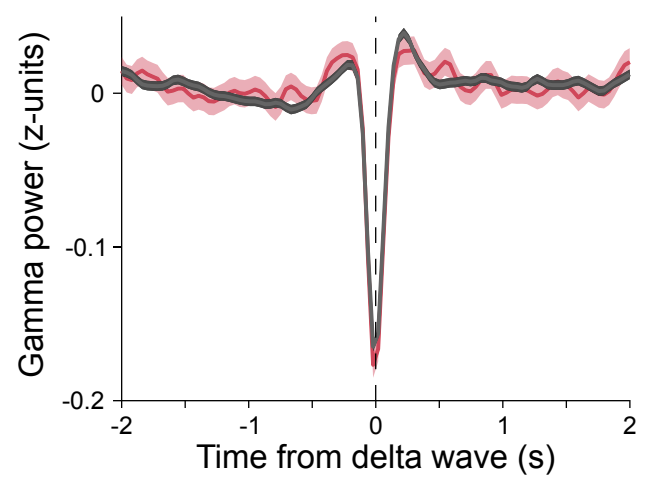

C

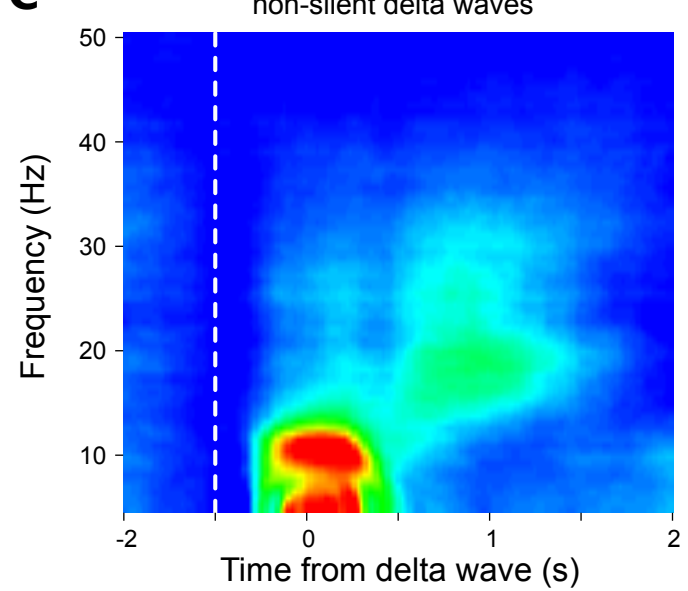

B

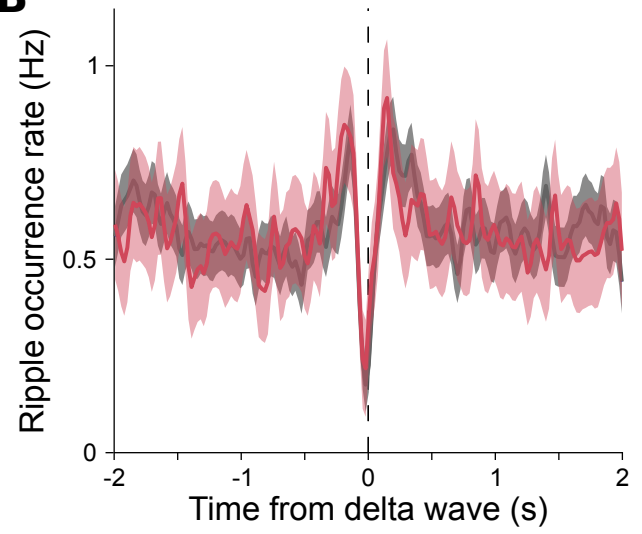

silent delta waves

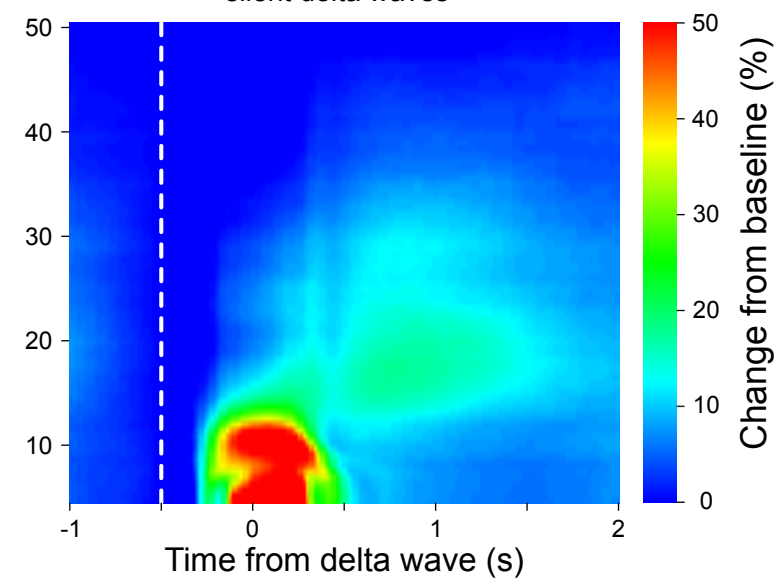

Fig. S5. Characteristics of silent and non-silent delta waves. (A) Gamma power (zscored; curves and shaded areas, mean \pm s.e.m.) decreased around delta waves regardless of whether spikes were detected during the delta waves (non-silent, red) or not (silent, black). (B) Ripple occurrence rate (curves and shaded areas, mean \pm s.e.m.) preceding and following delta waves was similar for silent (black) and non-silent (red) delta waves. (C) Peri-event spectrograms centered on non-silent (left) and silent (right) delta waves, normalized relative to baseline power recorded $1 \mathrm{~s}$ before delta waves (dashed lines). Note the increase in spindleband activity following both non-silent and silent delta waves. 

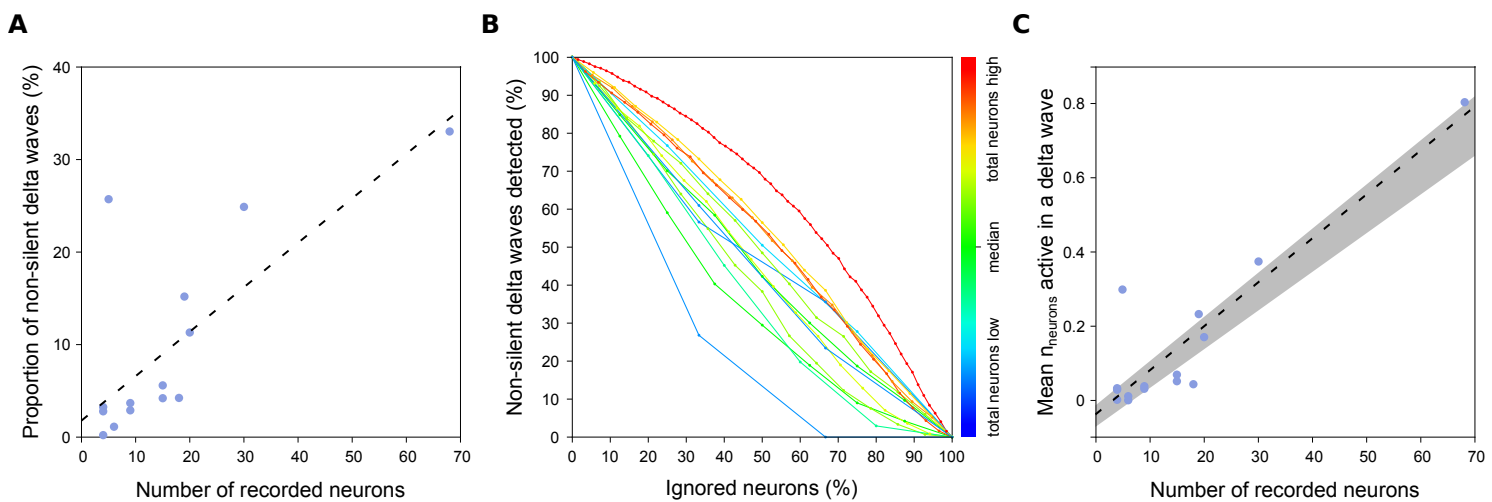

Fig. S6. Delta spike detection. (A) The proportion of non-silent delta waves increases with the number of recorded neurons (Pearson's $r=0.7456, P=0.0014$; dashed line, regression line). (B) For each session, delta waves were repeatedly detected using decreasing random subpopulations of neurons. Color-code: total number of neurons recorded in the session (range, 4-68). Note that the proportion of delta waves with detected delta spikes is greatly underestimated in limited data sets, and this effect is even stronger in sessions with fewer recorded neurons (cold colors). (C) The mean number of neurons active during a given delta wave linearly increases with the number of recorded neurons (Pearson's $r=0.9041, P<0.001$; dashed line, regression line). Shaded area: 95\% confidence intervals for the regression line obtained with jackknife resampling. Extrapolating to the total number of neurons in the mPFC (34), the number of neurons expected to fire in any given delta wave would be $3800-4600$. 

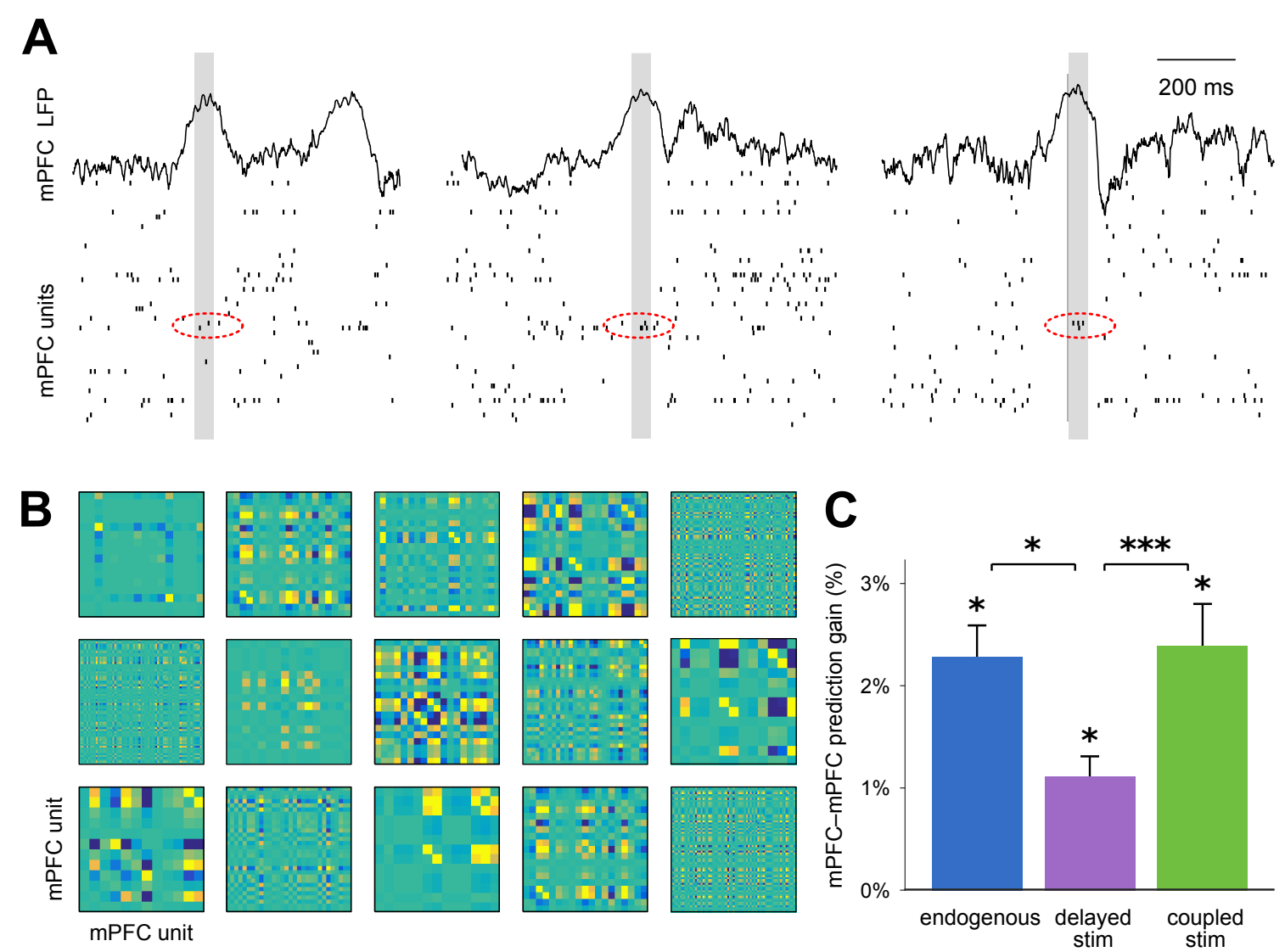

Fig. S7. Activity components during delta waves. (A) Examples of correlated firing during delta waves (red circles). (B) Example significant templates (putative cell assemblies) identified using independent component analysis (ICA). (C) Prediction gain (bars: mean \pm s.e.m.) of a generalized linear model (GLM) trained to predict the delta spikes of each prefrontal neuron, based on the delta spikes of all other prefrontal neurons (peer activity) for endogenous as well as induced delta waves (see Fig. 4). Prefrontal peer activity substantially improved the prediction gain compared to a control condition where delta spikes were predicted based solely on overall delta activity levels, i.e. summing spikes from all peers and ignoring cell identities, for both endogenous $(P=0.0028$, Wilcoxon signed rank test) and induced delta waves (delayed stimulation: $P<0.001$; coupled stimulation: $P<0.001$, Wilcoxon signed rank tests). Prediction gain was higher in endogenous delta waves and induced coupled delta waves than in induced delayed delta waves $(P=0.0258$, Kruskal-Wallis test; endogenous vs delayed stimulation: $P=0.0221$; coupled vs delayed stimulation: $P<0.001$; endogenous vs delayed stimulation: $P=0.4377$, Wilcoxon rank-sum tests). 
Rat 199

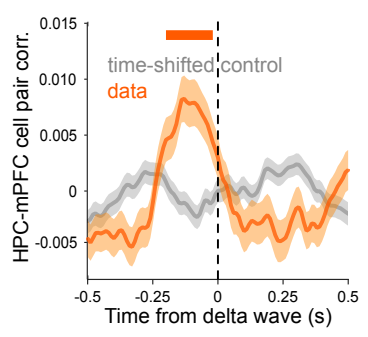

Rat 220

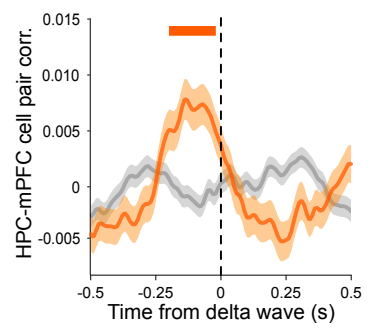

Rat 211

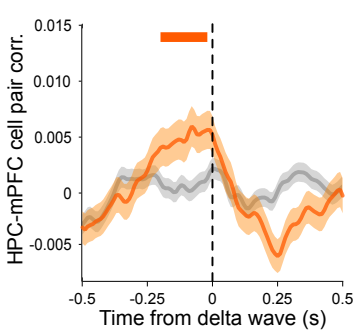

Rat 221

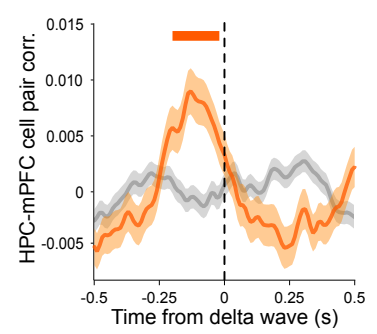

Rat 214

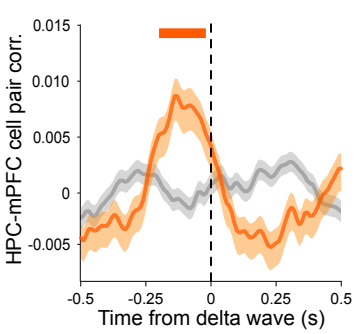

Rat 230

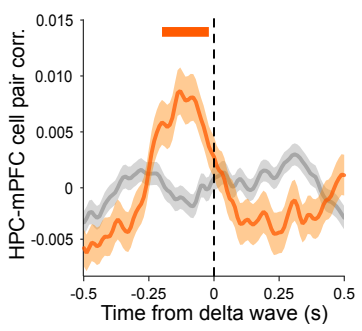

Rat 219

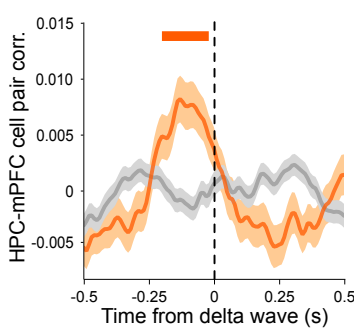

Rat 229

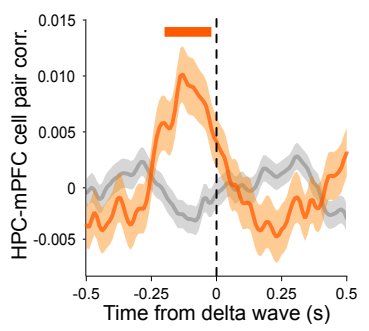

Fig. S8. Hippocampal prediction of delta spikes does not critically depend on data recorded from any single rat. Cross-correlations (curves and shaded areas, mean \pm s.e.m.) between hippocampal ripple activity (sliding window) and delta spikes (fixed, $0 \mathrm{~s}$ ). Observed cross-correlations (orange) vs time-shifted control cross-correlations (grey) for hippocampal ripple activity preceding delta waves (horizontal orange line: Monte-Carlo test, $P<0.05$ ). Each panel repeats the analysis presented in Fig. 2 leaving one rat out. 

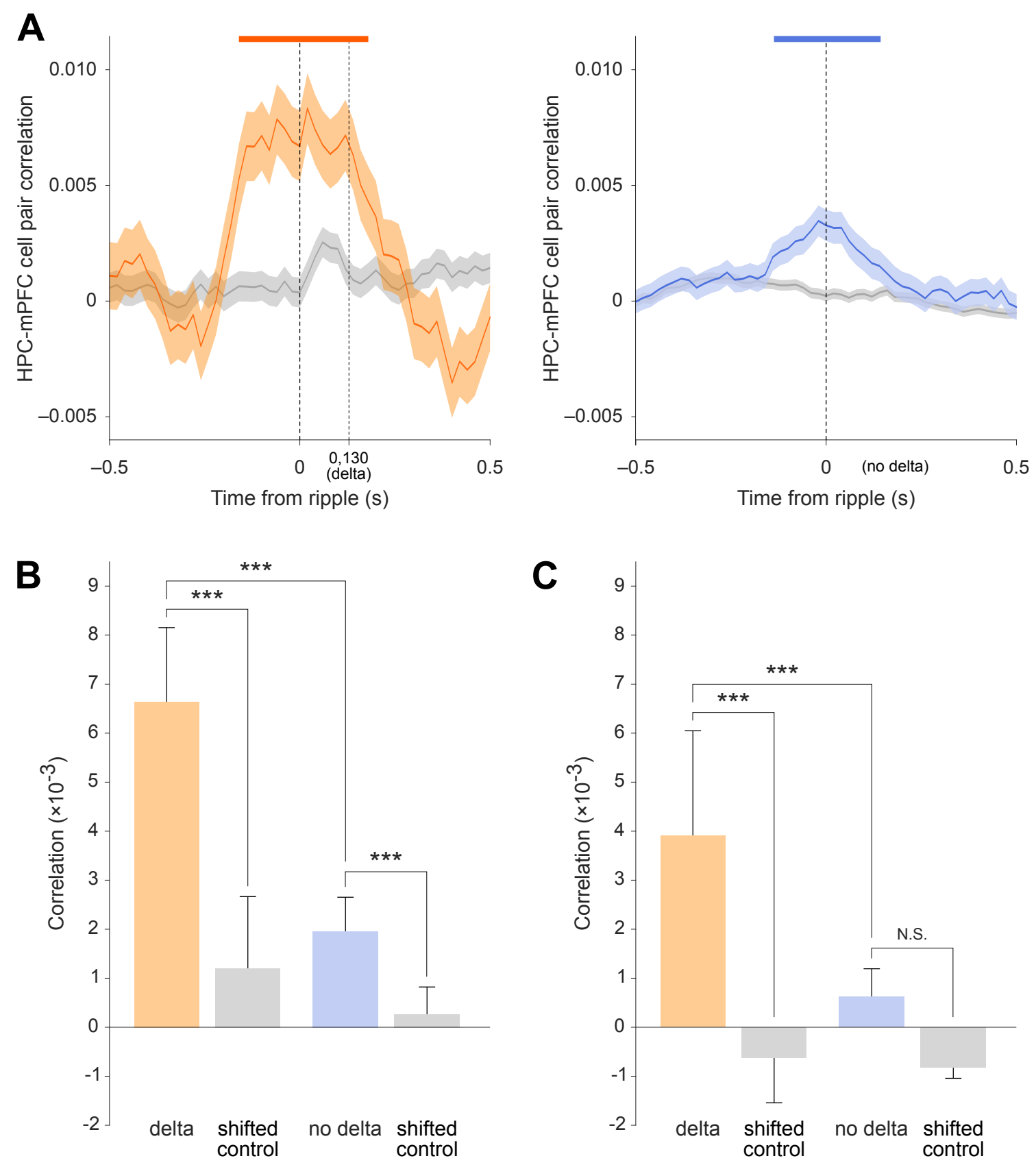

Fig. S9. Hippocampal ripple activity better predicts cortical spikes in the presence of a delta wave. (A) Cross-correlations (curves and shaded areas, mean \pm s.e.m.) between hippocampal ripple activity (fixed, $0 \mathrm{~s}$ ) and cortical spikes (sliding window), in the presence (left) or absence (right) of a delta wave following (within $200 \mathrm{~ms}$ ) the ripple. Observed crosscorrelations (orange, blue) vs time-shifted control cross-correlations (grey) (horizontal orange and blue lines: Monte-Carlo tests, $P<0.05)$. (B) Correlation between hippocampal ripple activity and subsequent cortical spikes $([0,200]$ ms time window) in the presence (orange bar) or absence (blue bar) of delta waves (grey bars, time-shifted control correlations). ${ }^{* * *} P<0.001$, Wilcoxon signed rank tests. (C) Same as (B) but using a more stringent $30 \mathrm{~ms}$ window centered on the delta wave following the ripple (orange) or at $130 \mathrm{~ms}$ post ripple (blue). ${ }^{* * *} P<0.001$, Wilcoxon signed rank tests. 


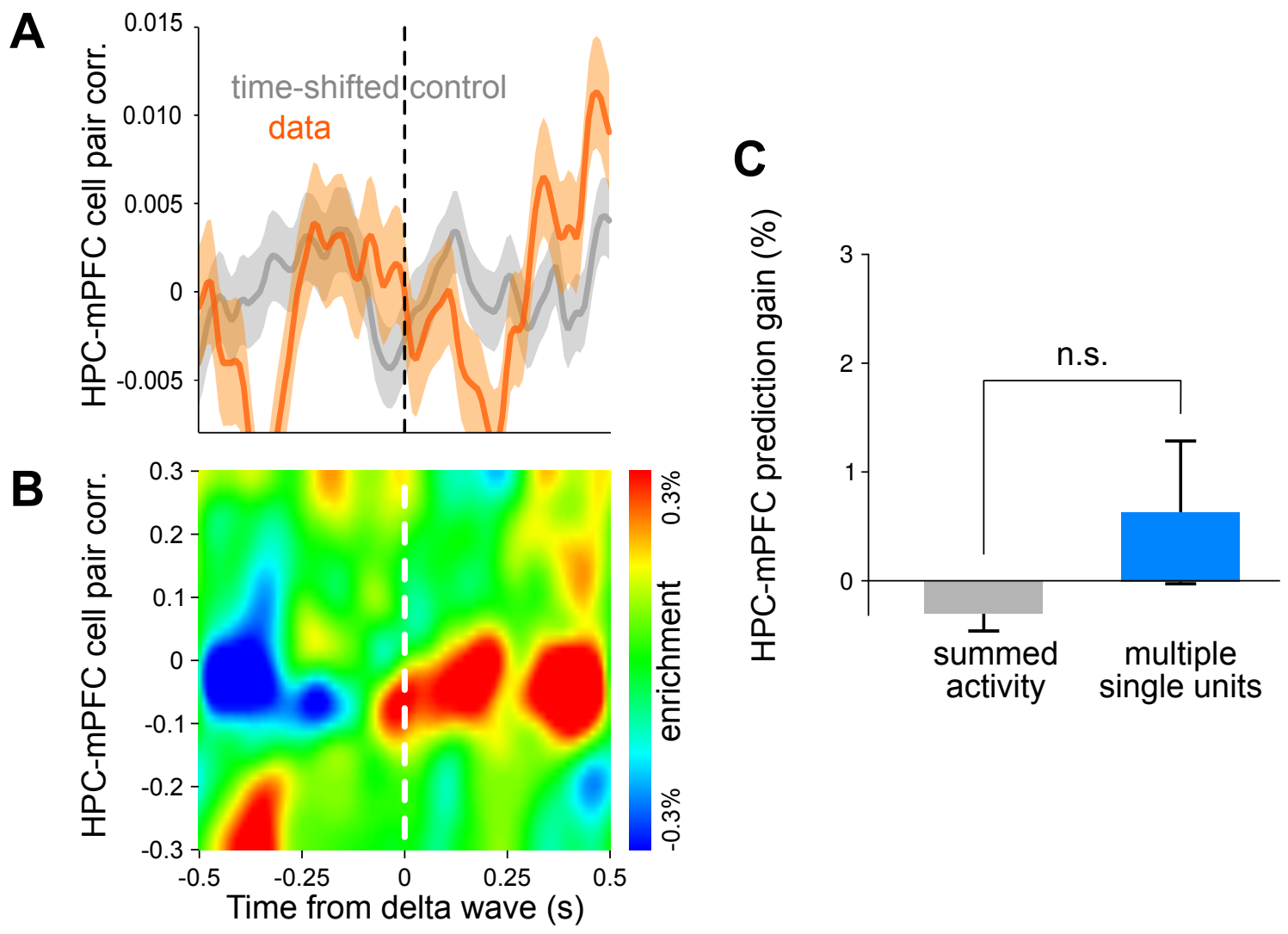

Fig. S10. Hippocampal ripple activity fails to predict delta spikes in sleep preceding behavior. (A) Cross-correlations (curves and shaded areas, mean \pm s.e.m.) between hippocampal ripple activity (sliding window) and delta spikes (fixed, $0 \mathrm{~s}$ ) in sleep before the task. Observed cross-correlations (orange) were not significantly different from time-shifted control cross-correlations (grey) (Monte-Carlo test, $P>0.05$ ). (C) Comparative distribution between data and control in (A). There was no enrichment of positively correlated pairs of hippocampal ripple activity and subsequent delta waves in sleep before the task. (C) Performance of a GLM trained to predict prefrontal activity during delta waves in sleep before the task based on preceding hippocampal ripple activity ( $200 \mathrm{~ms}$ window), measured as percent improvement relative to a shuffled control (prediction gain). Multiple single-unit hippocampal activity did not improve performance relative to global hippocampal drive ignoring cell identity $(P=0.6251$, Wilcoxon signed rank test). Neither model could significantly predict prefrontal activity during delta waves in sleep before the task (multiple single units, $P=0.3640$; summed activity, $P=1$; Wilcoxon rank-sum tests). 
A

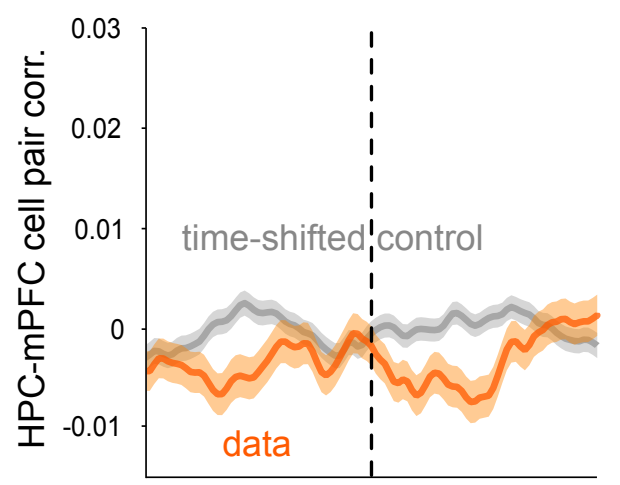

B

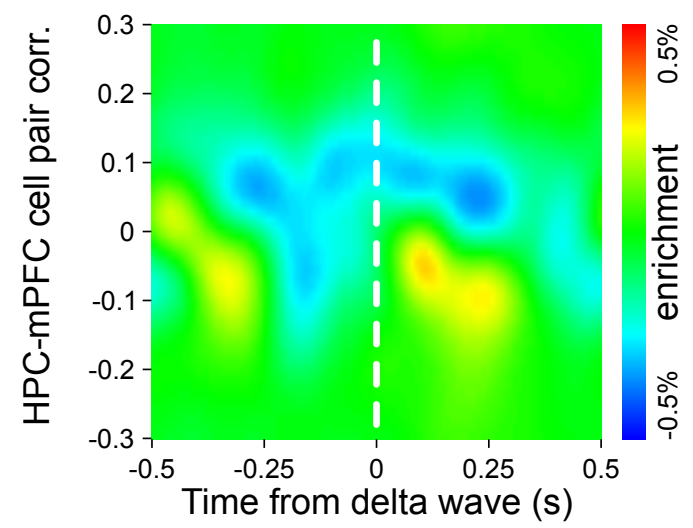

C

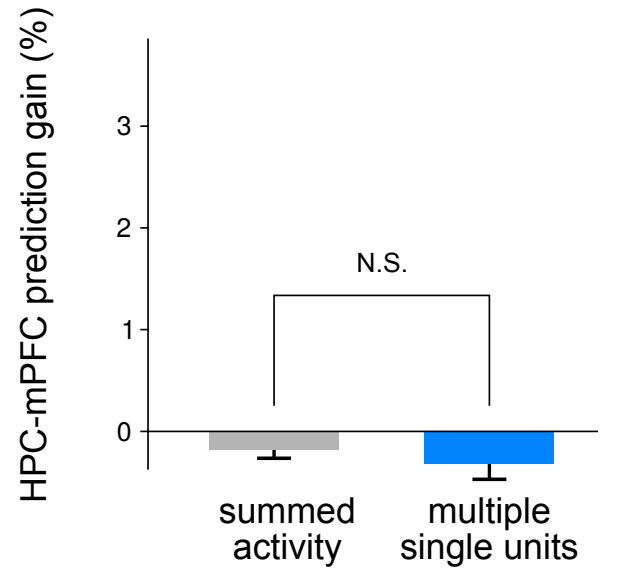

Partner mPFC cells $(n=57)$
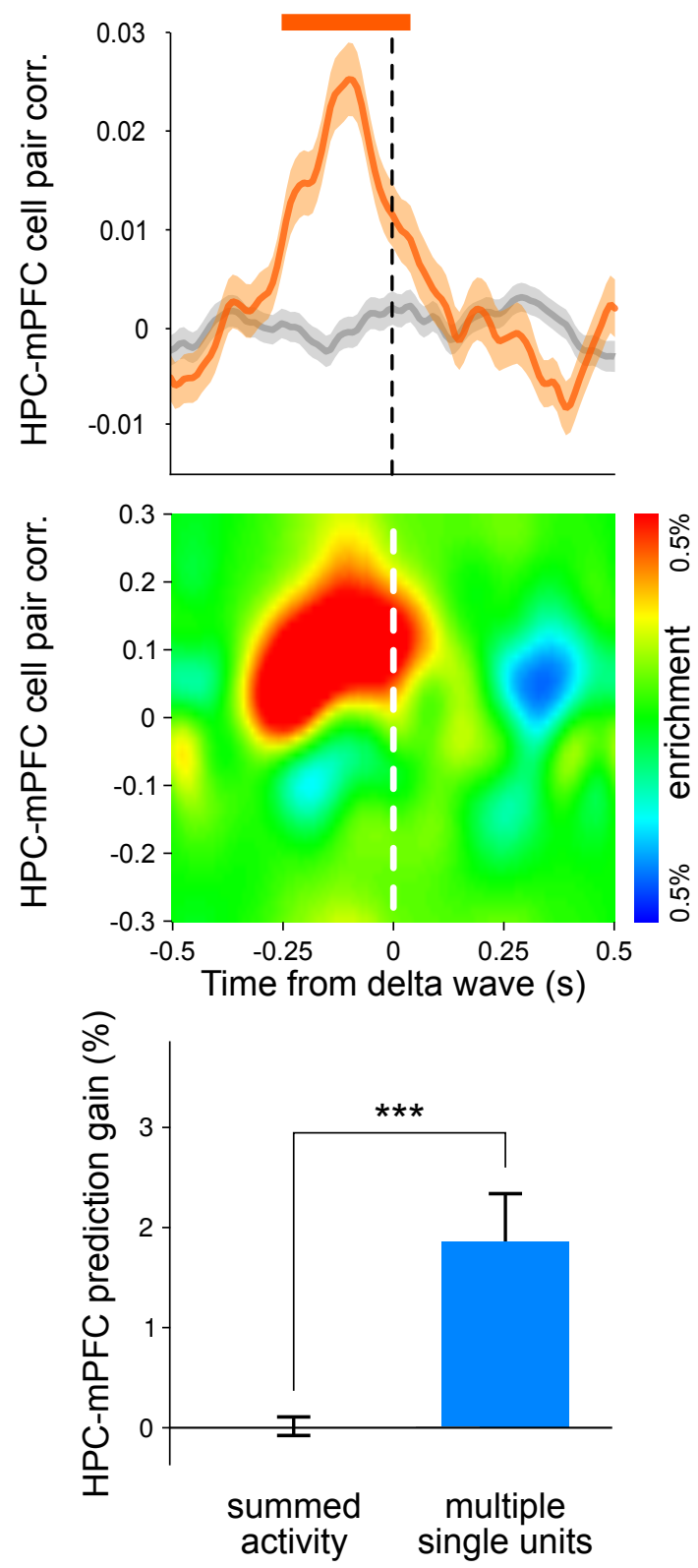

Fig. S11. Hippocampal ripple activity predicts delta spikes. (A) Cross-correlations (curves and shaded areas, mean \pm s.e.m.) between hippocampal ripple activity (sliding window) and delta spikes (fixed, $0 \mathrm{~s}$ ) separately for non-partner cells (left) vs partner cells (right). Observed cross-correlations (orange) vs time-shifted control cross-correlations (grey) for hippocampal ripple activity preceding delta waves (horizontal orange line: Monte-Carlo test, $P<0.05)$. Hippocampal spikes could predict delta spikes only for partner cells (right). (B) Enrichment in positive correlations (comparative distribution between data and control in (A)) when hippocampal activity was correlated to subsequent prefrontal delta spikes. (C) Performance of a GLM trained to predict prefrontal activity during delta waves based on preceding hippocampal ripple activity ( $200 \mathrm{~ms}$ window), measured as percent improvement relative to a shuffled control (prediction gain). Delta partner spikes were significantly predicted by multiple single-unit hippocampal activity $(P<0.001$, Wilcoxon rank-sum tests), but not by global hippocampal drive ignoring cell identity (non-partner hippocampal units, $P=1$, Wilcoxon rank-sum test). ${ }^{* * *} P<0.001$, Wilcoxon signed rank tests. 

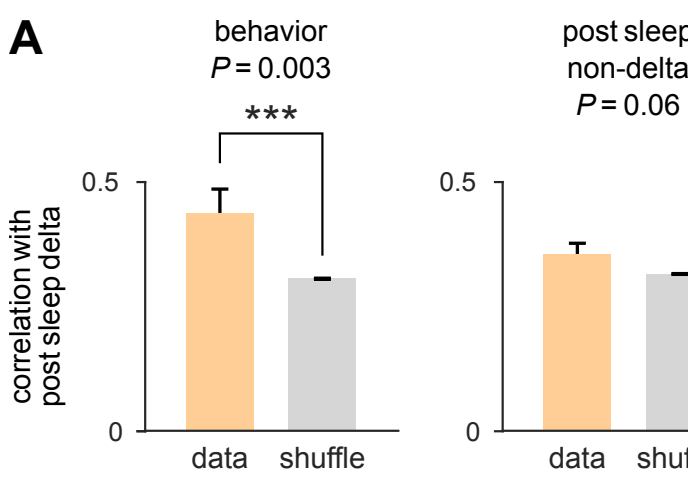

pre sleep
non-delta

pre sleep

delta

$P=0.09$

$P=0.40$

B behavior
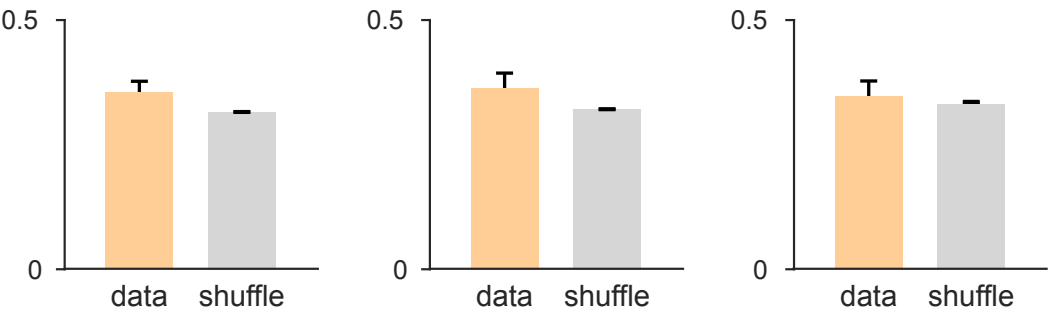

$$
\begin{gathered}
\text { pre sleep } \\
\text { non-delta } \\
P=0.73
\end{gathered}
$$

post sleep

non-delta

$P=0.99$
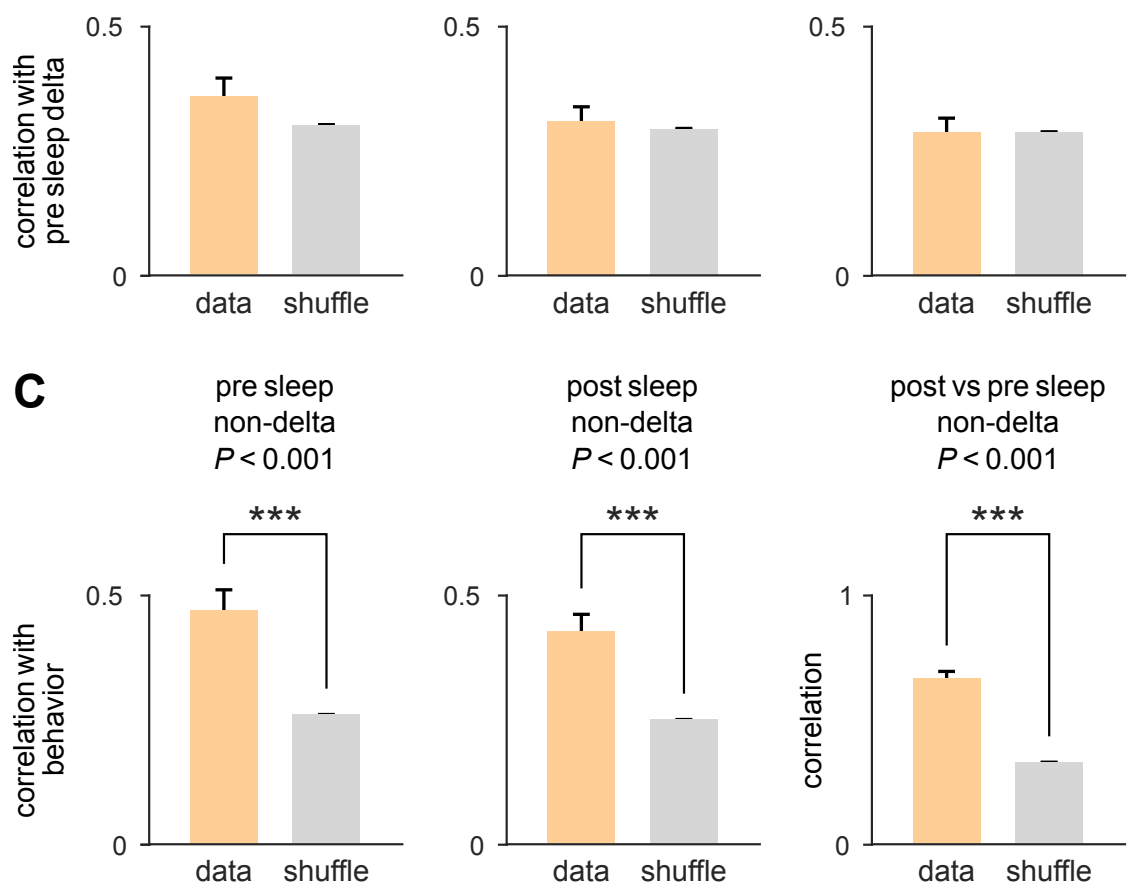

Fig. S12. Delta assemblies are uniquely expressed during task performance. Comparison of assemblies during delta waves and non-delta periods in sleep preceding behavior (pre-sleep), during behavior, and during delta waves and non-delta periods in sleep following behavior (post-sleep). (A) Comparison of post-sleep delta assemblies and all other assemblies (orange: correlation between assemblies; grey: shuffled controls). Correlations were significant only between delta assemblies and assemblies expressed during behavior. (B) Comparison of pre-sleep delta assemblies and other assemblies. No correlation was significant. (C) Comparison of non-delta assemblies. Assemblies expressed during behavior were significantly correlated with both post- and pre-sleep assemblies, consistent with similar findings about hippocampal ripple activity during sleep. 

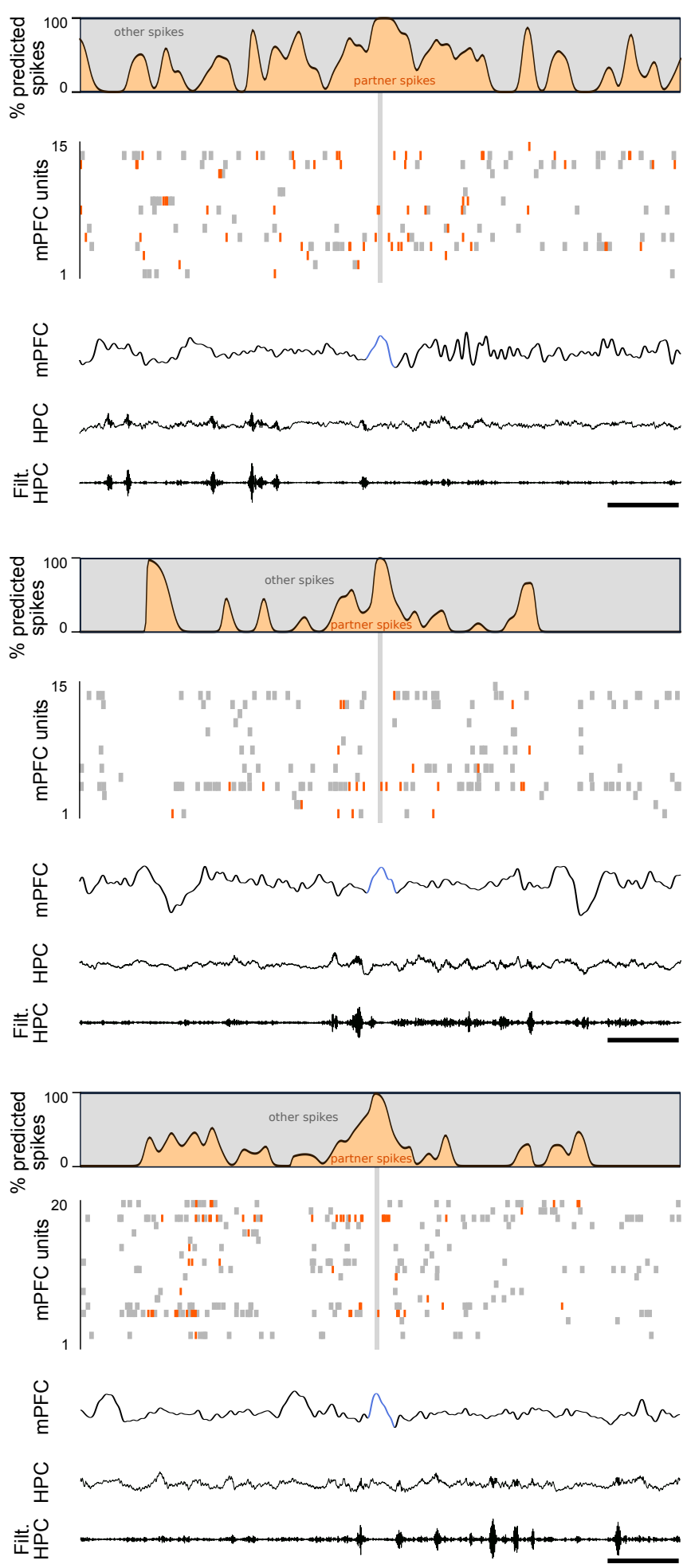

Fig. S13. Delta waves isolate cortical computations. Simultaneous recordings of prefrontal and hippocampal activity around delta waves (grey shaded rectangles). Top: proportion of prefrontal spikes predicted by the firing of hippocampal cells (partner spikes). Center: raster plot of spikes emitted by prefontal units (red ticks: partner spikes, grey ticks: other spikes). Bottom: simultaneously recorded local field potentials in the mPFC (blue: delta wave) and hippocampus (broadband and ripple-band filtered signal). Black calibration bars: $0.5 \mathrm{~s}$. 

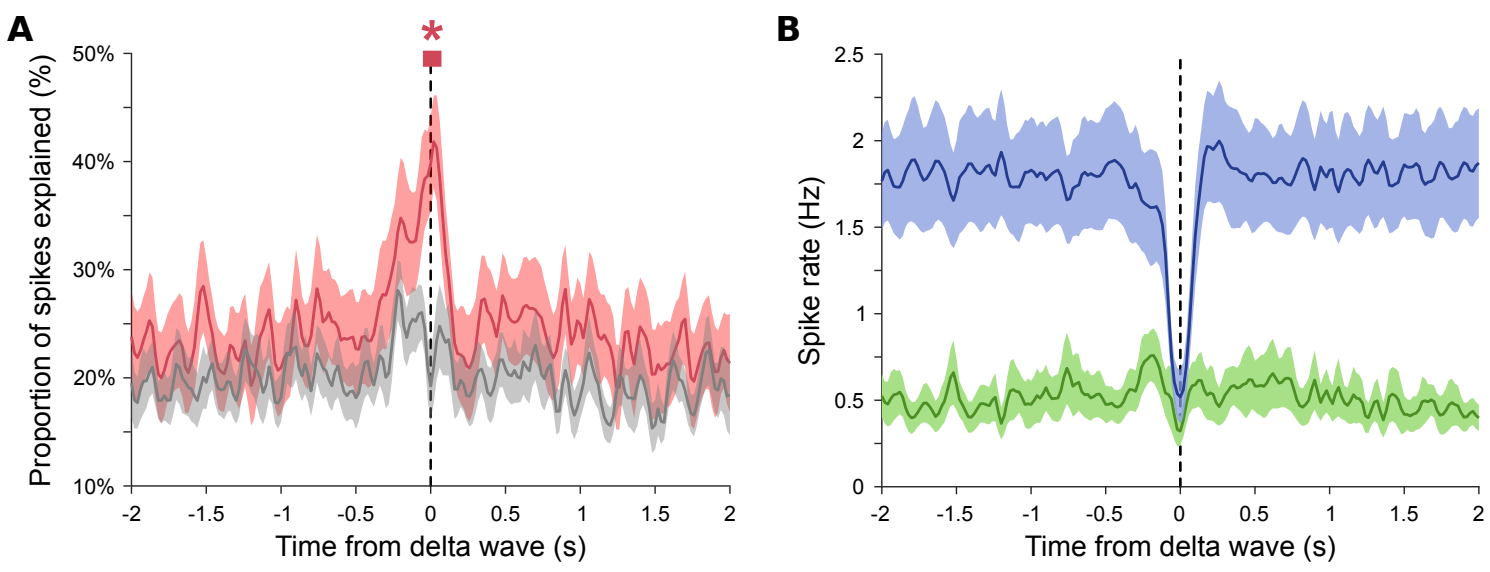

Fig. S14. Selective silencing of cortical spikes during delta waves. (A) Proportion of prefrontal spikes explained by the preceding hippocampal activity (partner spikes). Observed proportions (red) were significantly different from time-shifted controls (grey), precisely during delta waves (curves and shaded areas, mean \pm s.e.m.; horizontal red line: Monte-Carlo test, $P<0.05$ ). (B) Average rate (curves and shaded areas, mean \pm s.e.m.) of prefrontal partner spikes (green) and other spikes (blue) around delta waves. Note that the firing rate of partner spikes remains relatively constant, whereas the firing rate of other spikes decreases dramatically at $t=0$, effectively boosting the signal-to-noise ratio during delta waves.

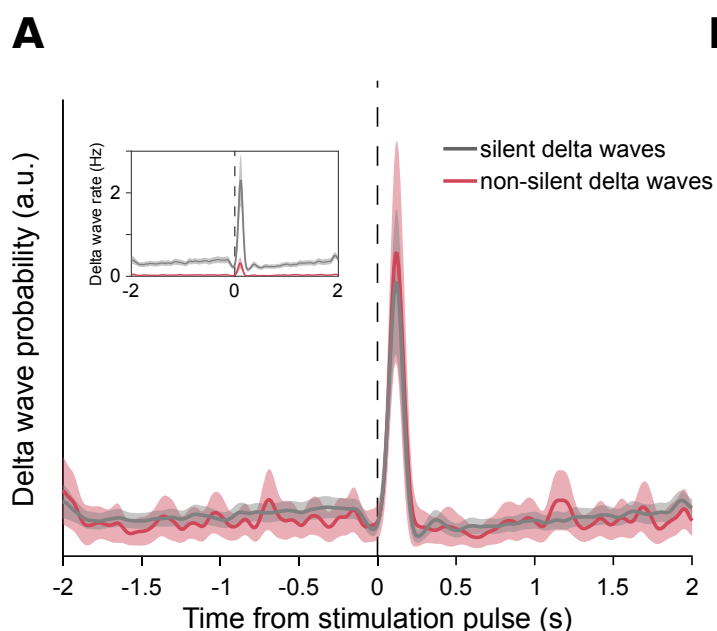

B

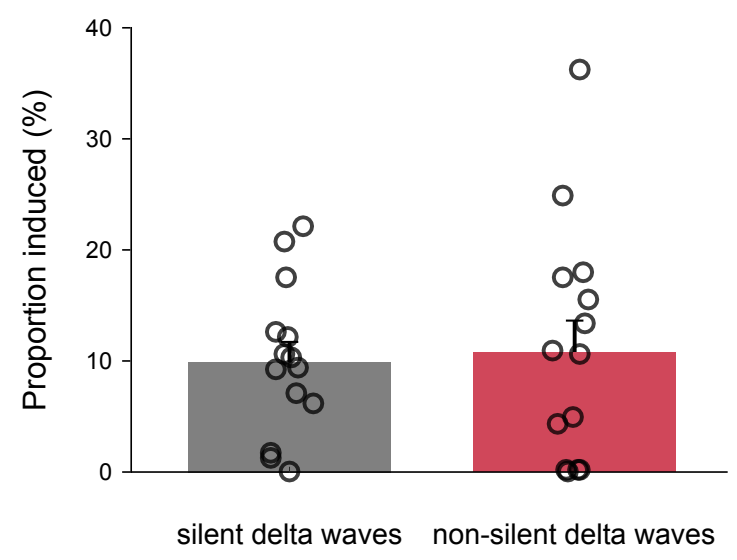

Fig. S15. Cortical stimulation triggers both silent and non-silent delta waves. (A) Occurrence rate (mean \pm s.e.m.) for silent (grey) and non-silent (red) delta waves following cortical stimulation. Rate is normalized by the total number of silent and non-silent delta waves, respectively (inset: non-normalized rates). (B) Proportion of delta waves following stimulation pulses within $200 \mathrm{~ms}$ (bars: median \pm s.e.median; open circles: individual sessions) was not different between silent (black) and non-silent (red) delta waves $(P>0.05$, Wilcoxon signed rank test). 
mPFC units

mPFC units

\begin{tabular}{cccccc} 
session & delta waves & mPFC units & HPC units & with HPC partners & with mPFC peers \\
\hline 1 & 1150 & 19 & 10 & 8 & 17 \\
2 & 1665 & 67 & 10 & 9 & 67 \\
3 & 794 & 9 & 10 & 0 & 0 \\
4 & 482 & 1 & 9 & 0 & 0 \\
5 & 381 & 6 & 13 & 0 & 5 \\
6 & 1512 & 13 & 8 & 5 & 5 \\
7 & 1579 & 8 & 4 & 4 & 0 \\
8 & 595 & 5 & 4 & 0 & 2 \\
9 & 216 & 1 & 3 & 0 & 0 \\
10 & 1360 & 12 & 13 & 5 & 9 \\
11 & 2046 & 28 & 6 & 6 & 27 \\
12 & 358 & 4 & 14 & 1 & 0 \\
13 & 1910 & 19 & 23 & 11 & 6 \\
14 & 3186 & 10 & 12 & 1 &
\end{tabular}

Table S1. Number of units recorded from the hippocampus and prefrontal cortex during slow wave sleep. Only units recorded on channels where delta waves could be detected were included. 


\begin{tabular}{|c|c|c|c|c|c|c|c|c|c|}
\hline session & unit & delta spikes & $\begin{array}{c}\mathrm{HPC} \\
\text { partners }\end{array}$ & $\begin{array}{c}\mathrm{mPFC} \\
\text { peers }\end{array}$ & session & unit & delta spikes & $\begin{array}{c}\mathrm{HPC} \\
\text { partners }\end{array}$ & $\begin{array}{c}\mathrm{mPFC} \\
\text { peers }\end{array}$ \\
\hline 1 & 1 & 3 & 0 & 1 & . & & & 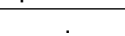 & \\
\hline 1 & 2 & 11 & 0 & 4 & : & $:$ & $:$ & : & $:$ \\
\hline 1 & 3 & 3 & 0 & 7 & 2 & 41 & 51 & 0 & 16 \\
\hline 1 & 4 & 25 & 0 & 6 & 2 & 42 & 1 & 0 & 2 \\
\hline 1 & 5 & 38 & 2 & 6 & 2 & 43 & 13 & 0 & 20 \\
\hline 1 & 6 & 13 & 2 & 4 & 2 & 44 & 1 & 0 & 4 \\
\hline 1 & 7 & 5 & 0 & 0 & 2 & 45 & 9 & 0 & 9 \\
\hline 1 & 8 & 1 & 0 & 0 & 2 & 46 & 74 & 1 & 17 \\
\hline 1 & 9 & 11 & 0 & 3 & 2 & 47 & 2 & 0 & 7 \\
\hline 1 & 10 & 5 & 1 & 5 & 2 & 48 & 5 & 0 & 9 \\
\hline 1 & 11 & 17 & 3 & 6 & 2 & 49 & 6 & 0 & 3 \\
\hline $\begin{array}{l}1 \\
1\end{array}$ & $\begin{array}{l}11 \\
12\end{array}$ & $\begin{array}{c}11 \\
4\end{array}$ & 2 & 3 & 2 & 50 & 40 & 2 & 26 \\
\hline 1 & 13 & 20 & 0 & 11 & 2 & 51 & 35 & 0 & 20 \\
\hline 1 & 14 & 4 & 1 & $\begin{array}{c}11 \\
5\end{array}$ & 2 & 52 & 3 & 0 & 1 \\
\hline 1 & 15 & 1 & 1 & 2 & 2 & 53 & 88 & 0 & 18 \\
\hline 1 & 16 & 8 & 0 & 7 & 2 & 54 & 93 & 0 & 31 \\
\hline 1 & 17 & 52 & 1 & 11 & 2 & 55 & 4 & 0 & 13 \\
\hline 1 & 18 & 13 & 0 & 2 & 2 & 56 & 10 & 0 & 6 \\
\hline 1 & 19 & 18 & 0 & 3 & 2 & 57 & 8 & 1 & 6 \\
\hline 2 & 1 & 16 & 0 & 15 & 2 & 58 & 28 & 0 & 14 \\
\hline 2 & 2 & 66 & 0 & 16 & 2 & 59 & 20 & 0 & 13 \\
\hline 2 & 3 & 43 & 0 & 21 & 2 & 60 & 24 & 0 & 9 \\
\hline 2 & 4 & 45 & 0 & 16 & 2 & 61 & 10 & 0 & 11 \\
\hline 2 & 5 & 8 & 0 & 7 & 2 & 62 & 5 & 0 & 3 \\
\hline 2 & 6 & 14 & 0 & 6 & 2 & 63 & 11 & 0 & 10 \\
\hline 2 & 7 & 14 & 0 & 5 & 2 & 64 & 8 & 4 & 10 \\
\hline 2 & 8 & 55 & 0 & 12 & 2 & 65 & 18 & 0 & 13 \\
\hline 2 & 9 & 7 & 0 & 4 & 2 & 66 & 8 & 0 & 6 \\
\hline 2 & 10 & 2 & 0 & 10 & 2 & 67 & 25 & 1 & 8 \\
\hline 2 & 11 & 39 & 2 & 21 & 3 & 1 & 1 & 0 & 0 \\
\hline 2 & 12 & 3 & 0 & 11 & 3 & 2 & 2 & 0 & 0 \\
\hline 2 & 13 & 80 & 1 & 30 & 3 & 3 & 1 & 0 & 0 \\
\hline 2 & 14 & 34 & 0 & 21 & 3 & 4 & 2 & 0 & 0 \\
\hline 2 & 15 & 6 & 0 & 7 & 3 & 5 & 3 & 0 & 0 \\
\hline 2 & 16 & 3 & 0 & 10 & 3 & 6 & 2 & 0 & 0 \\
\hline 2 & 17 & 57 & 0 & 23 & 3 & 7 & 10 & 0 & 0 \\
\hline 2 & 18 & 23 & 0 & 9 & 3 & 8 & 1 & 0 & 0 \\
\hline 2 & 19 & 3 & 0 & 7 & 3 & 9 & 3 & 0 & 0 \\
\hline 2 & 20 & 2 & 0 & 10 & 4 & 1 & 1 & 0 & 0 \\
\hline 2 & 21 & 17 & 0 & 4 & 5 & 1 & 2 & 0 & 0 \\
\hline 2 & 22 & 2 & 0 & 8 & 5 & 2 & 3 & 0 & 1 \\
\hline 2 & 23 & 11 & 0 & 4 & 5 & 3 & 3 & 0 & 1 \\
\hline 2 & 24 & $\begin{array}{c}11 \\
6\end{array}$ & 0 & 6 & 5 & 4 & 4 & 0 & 1 \\
\hline 2 & 25 & 3 & 0 & 6 & 5 & 5 & 6 & 0 & 2 \\
\hline 2 & 26 & 6 & 0 & 6 & 5 & 6 & 2 & 0 & 1 \\
\hline 2 & 27 & 2 & 1 & 7 & 6 & 1 & 1 & 0 & 0 \\
\hline 2 & 28 & 21 & 0 & 10 & 6 & 2 & 1 & 0 & 1 \\
\hline 2 & 29 & 23 & 0 & 4 & 6 & 3 & 2 & 0 & 0 \\
\hline 2 & 30 & 17 & 0 & 13 & 6 & 4 & 7 & 1 & 0 \\
\hline 2 & 31 & 20 & 0 & 14 & 6 & 5 & 7 & 0 & 0 \\
\hline 2 & 32 & 1 & 0 & 3 & 6 & 6 & 5 & 0 & 1 \\
\hline 2 & 33 & 14 & 0 & 15 & 6 & 7 & 6 & 2 & 0 \\
\hline 2 & 34 & 2 & 0 & 12 & 6 & 8 & 10 & 0 & 0 \\
\hline 2 & 35 & 5 & 0 & 8 & 6 & 9 & 1 & 0 & 1 \\
\hline 2 & 36 & 1 & 0 & 1 & 6 & 10 & 7 & 2 & 2 \\
\hline 2 & 37 & 4 & 0 & 4 & 6 & 11 & 8 & 1 & 0 \\
\hline 2 & 38 & 2 & 1 & 5 & 6 & 12 & 8 & 1 & 0 \\
\hline 2 & 39 & 53 & 0 & 18 & 6 & 13 & 4 & 0 & 1 \\
\hline 2 & 40 & 7 & 0 & 2 & 7 & 1 & 20 & 0 & 0 \\
\hline$\vdots$ & $\vdots$ & $\vdots$ & $\vdots$ & $\vdots$ & $\vdots$ & $\vdots$ & $\vdots$ & $\vdots$ & \\
\hline
\end{tabular}

Table S2. Number of delta spikes emitted by prefrontal cortical cells during slow wave sleep. 


\begin{tabular}{|c|c|c|c|c|c|c|c|c|c|}
\hline session & unit & delta spikes & $\begin{array}{c}\text { HPC } \\
\text { partners }\end{array}$ & $\begin{array}{c}\text { mPFC } \\
\text { peers }\end{array}$ & session & unit & delta spikes & $\begin{array}{c}\mathrm{HPC} \\
\text { partners }\end{array}$ & $\begin{array}{c}\mathrm{mPFC} \\
\text { peers }\end{array}$ \\
\hline$\vdots$ & $\vdots$ & $\vdots$ & $\vdots$ & $\vdots$ & $\vdots$ & $\vdots$ & $\vdots$ & $\vdots$ & $\vdots$ \\
\hline 7 & 2 & 5 & 1 & 0 & 11 & 18 & 18 & 0 & 5 \\
\hline 7 & 3 & 3 & 0 & 0 & 11 & 19 & 17 & 0 & 1 \\
\hline 7 & 4 & 1 & 0 & 0 & 11 & 20 & 12 & 0 & 3 \\
\hline 7 & 5 & 1 & 0 & 0 & 11 & 21 & 41 & 0 & 3 \\
\hline 7 & 6 & 23 & 1 & 0 & 11 & 22 & 14 & 0 & 6 \\
\hline 7 & 7 & 4 & 1 & 0 & 11 & 23 & 104 & 0 & 7 \\
\hline 7 & 8 & 4 & 2 & 0 & 11 & 24 & 9 & 0 & 4 \\
\hline 8 & 1 & 12 & 0 & 0 & 11 & 25 & 16 & 0 & 4 \\
\hline 8 & 2 & 4 & 0 & 1 & 11 & 26 & 49 & 1 & 5 \\
\hline 8 & 3 & 50 & 0 & 0 & 11 & 27 & 14 & 0 & 7 \\
\hline 8 & 4 & 38 & 0 & 0 & 11 & 28 & 10 & 1 & 1 \\
\hline 8 & 5 & 74 & 0 & 1 & 12 & 1 & 1 & 1 & 0 \\
\hline 9 & 1 & 7 & 0 & 0 & 12 & 2 & 1 & 0 & 0 \\
\hline 10 & 1 & 3 & 0 & 0 & 12 & 3 & 1 & 0 & 0 \\
\hline 10 & 2 & 3 & 1 & 1 & 12 & 4 & 1 & 0 & 0 \\
\hline 10 & 3 & 27 & 1 & 0 & 13 & 1 & 18 & 1 & 5 \\
\hline 10 & 4 & 4 & 2 & 5 & 13 & 2 & 8 & 0 & 2 \\
\hline 10 & 5 & 9 & 1 & 4 & 13 & 3 & 92 & 1 & 6 \\
\hline 10 & 6 & 10 & 1 & 2 & 13 & 4 & 16 & 0 & 5 \\
\hline 10 & 7 & 1 & 0 & 1 & 13 & 5 & 3 & 3 & 1 \\
\hline 10 & 8 & 1 & 0 & 0 & 13 & 6 & 10 & 0 & 10 \\
\hline 10 & 9 & 3 & 0 & 1 & 13 & 7 & 9 & 2 & 4 \\
\hline 10 & 10 & 15 & 0 & 4 & 13 & 8 & 25 & 0 & 10 \\
\hline 10 & 11 & 15 & 0 & 4 & 13 & 9 & 69 & 1 & 9 \\
\hline 10 & 12 & 4 & 0 & 2 & 13 & 10 & 28 & 0 & 9 \\
\hline 11 & 1 & 27 & 0 & 0 & 13 & 11 & 27 & 3 & 7 \\
\hline 11 & 2 & 46 & 0 & 2 & 13 & 12 & 6 & 0 & 0 \\
\hline 11 & 3 & 10 & 0 & 8 & 13 & 13 & 7 & 1 & 3 \\
\hline 11 & 4 & 12 & 0 & 1 & 13 & 14 & 21 & 0 & 3 \\
\hline 11 & 5 & 11 & 0 & 2 & 13 & 15 & 14 & 1 & 4 \\
\hline 11 & 6 & 19 & 0 & 4 & 13 & 16 & 7 & 1 & 3 \\
\hline 11 & 7 & 8 & 0 & 3 & 13 & 17 & 9 & 2 & 6 \\
\hline 11 & 8 & 16 & 0 & 3 & 13 & 18 & 45 & 0 & 7 \\
\hline 11 & 9 & 5 & 1 & 5 & 13 & 19 & 30 & 1 & 0 \\
\hline 11 & 10 & 67 & 0 & 5 & 14 & 1 & 157 & 0 & 2 \\
\hline 11 & 11 & 5 & 0 & 3 & 14 & 2 & 83 & 0 & 2 \\
\hline 11 & 12 & 19 & 0 & 8 & 14 & 3 & 93 & 0 & 2 \\
\hline 11 & 13 & 35 & 1 & 6 & 14 & 4 & 84 & 1 & 3 \\
\hline 11 & 14 & 39 & 0 & 4 & 14 & 5 & 39 & 0 & 0 \\
\hline 11 & 15 & 32 & 1 & 5 & 14 & 6 & 65 & 0 & 1 \\
\hline 11 & 16 & 41 & 1 & 3 & 14 & 7 & 24 & 0 & 0 \\
\hline 11 & 17 & 70 & 0 & 8 & 14 & 8 & 48 & 0 & 0 \\
\hline \multirow[b]{2}{*}{$\vdots$} & 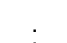 & 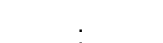 & . & . & 14 & 9 & 2 & 0 & 0 \\
\hline & : & : & : & . & 14 & 10 & 44 & 0 & 2 \\
\hline
\end{tabular}

Table S2 (cont.). Number of delta spikes emitted by prefrontal cortical cells during slow wave sleep. 